\title{
Une fouille en bordure des thermes de Jublains (Mayenne) : enfin un dodécaèdre en contexte archéologique!
}

Gérard Guillier, Richard Delage et Paul-André Besombes

\section{CpenEdition}

\section{Journals}

Édition électronique

URL : http://journals.openedition.org/rao/680

DOI : $10.4000 /$ rao.680

ISBN : 978-2-7535-1608-3

ISSN : 1775-3732

Éditeur

Presses universitaires de Rennes

\section{Édition imprimée}

Date de publication : 20 décembre 2008

Pagination : 269-289

ISBN : 978-2-7535-0789-0

ISSN : 0767-709X

Référence électronique

Gérard Guillier, Richard Delage et Paul-André Besombes, « Une fouille en bordure des thermes de Jublains (Mayenne) : enfin un dodécaèdre en contexte archéologique! », Revue archéologique de l'Ouest [En ligne], 25 | 2008, mis en ligne le 26 janvier 2011, consulté le 03 décembre 2020. URL : http:// journals.openedition.org/rao/680 ; DOI : https://doi.org/10.4000/rao.680 


\title{
Une fouille en bordure des thermes de Jublains (Mayenne) : enfin un dodécaèdre en contexte archéologique!
}

\author{
Excavation near the ancient baths of Jublains (Mayenne): \\ at last a dodecahedron in archaeological context!
}

\author{
Gérard GuillieR* et Richard Delage** \\ avec la collaboration de Paul-André Besombes***
}

Résumé : L’antique Nouiodunum (Jublains), capitale de cité des Diablintes, est une ville de plan orthonormé dont la parure monumentale, ainsi que quelques secteurs d'habitat et d'artisanat, sont relativement bien connus. En 1995, une intervention archéologique d'une centaine de mètres carrés a porté sur un îlot encore peu exploré du cœur de la ville. La rue antique $\mathrm{n}^{\circ} 8$ et ses abords ont été fouillés, confirmant notamment la période d'installation de la trame viaire, avant le règne de Flavius.

La mise au jour d'un petit bâtiment sur cave, incendié à la charnière des II $^{\mathrm{e}}$ et III $^{\mathrm{e}}$ siècles constitue une découverte remarquable, puisque les mobiliers recueillis montrent qu'il ne s'agit pas d'un simple habitat mais plutôt d'un lieu à vocation commerciale, peut-être spécialisé dans le négoce de denrées précieuses. Dans cette structure furent tout particulièrement recueillis un trébuchet et un dodécaèdre en bronze, objet énigmatique s'il en est dont les interprétations oscillent entre fonctions utilitaires et instrument de divination.

\begin{abstract}
The ancient city of Nouiodunum, now Jublains, is known for its monumental, relatively well-preserved thermae, temple and theatre. While several major excavations have been conducted there recently, an other one, in 1995, involved an area of about 100 square meters in the heart of the old city; it focused on an unexplored urban block in the centre of the town. The study of Street 8, running along the northern side of the thermae, led to the discovery of coins struck by Nero beneath the earliest level of the road. This find seems to confirm the theory of Nouiodunum's Via network installation at the beginning of the reign of Emporor Flavius.

A small building, drystone built over a cellar and destroyed by fire at the turning point of the IIt and III' centuries, seems to have had some commercial purpose. An enigmatic bronze dodecahedron was among the finds. All known items of this kind were unconnected discoveries, leading to speculations concerning their date and significance, from clearly utilitarian to quite mystical (divination) purposes. General consideration of the excavated structure suggests it was in the vicinity of a merchant's shop, which specialized in trade of precious goods: an other notable find is a balance, a really utilitarian item.
\end{abstract}

Mots clés : Mayenne, Jublains, Nouiodunum, Aulerques diablintes, rue antique, bâti sur sous-sol, céramique, monnaies, dodécaèdre, trébuchet.

Key words: Mayenne, Jublains, Nouiodunum, Aulerci Diablinti, Ancient street, building with cellar, ceramics, coins, dodecahadron, balance.

* Institut National de Recherches Archéologiques Préventives (INRAP Grand-Ouest, Cesson-Sévigné-Rennes).

** INRAP Grand-Ouest et UMR 8546 (CNRS/ENS, Paris).

*** Conservateur du Patrimoine, Service régional de l'Archéologie de Bretagne, Rennes. 


\section{INTRODUCTION}

Jublains, l'antique Nouiodunum, capitale de la cité des Aulerques Diablintes à l'époque gallo-romaine (fig. 1), est actuellement un petit bourg situé à $30 \mathrm{~km}$ au nord-nord-est de Laval, l'actuel chef-lieu du département de la Mayenne. Les nombreuses fouilles archéologiques qui y furent menées dès la fin du XviII ${ }^{\mathrm{e}}$ siècle, ont surtout mis en valeur la " forteresse " et la parure monumentale de la ville : sanctuaire, thermes et théâtre (Naveau [dir.], 1997), l'essentiel des quartiers urbains demeurant très largement méconnu. Ce n'est qu’à partir des découvertes de René Diehl, effectuées à la fin des années 1950, et grâce à la révélation de l'existence d'un plan antique (fig. 2) à trame orthogonale (Naveau, 1986), que plusieurs opérations, parfois d'importance, ont révélé tout le potentiel archéologique du site (Bocquet et al., 2004). Ces recherches ont également mis en évidence une occupation antérieure au développement de la ville romaine et permettent de replacer ce lieu dans le maillage des sites gaulois diablintes d'importance, avec ceux d'Entrammes et de Moulay.

C'est dans ce contexte archéologique particulièrement dense que le Service régional de l'Archéologie des Pays-dela-Loire a décidé de procéder, au cours de l'été 1995, à une opération de fouille préventive nécessitée par l'urgence : des travaux d'agrandissement de l'actuelle mairie, ainsi que

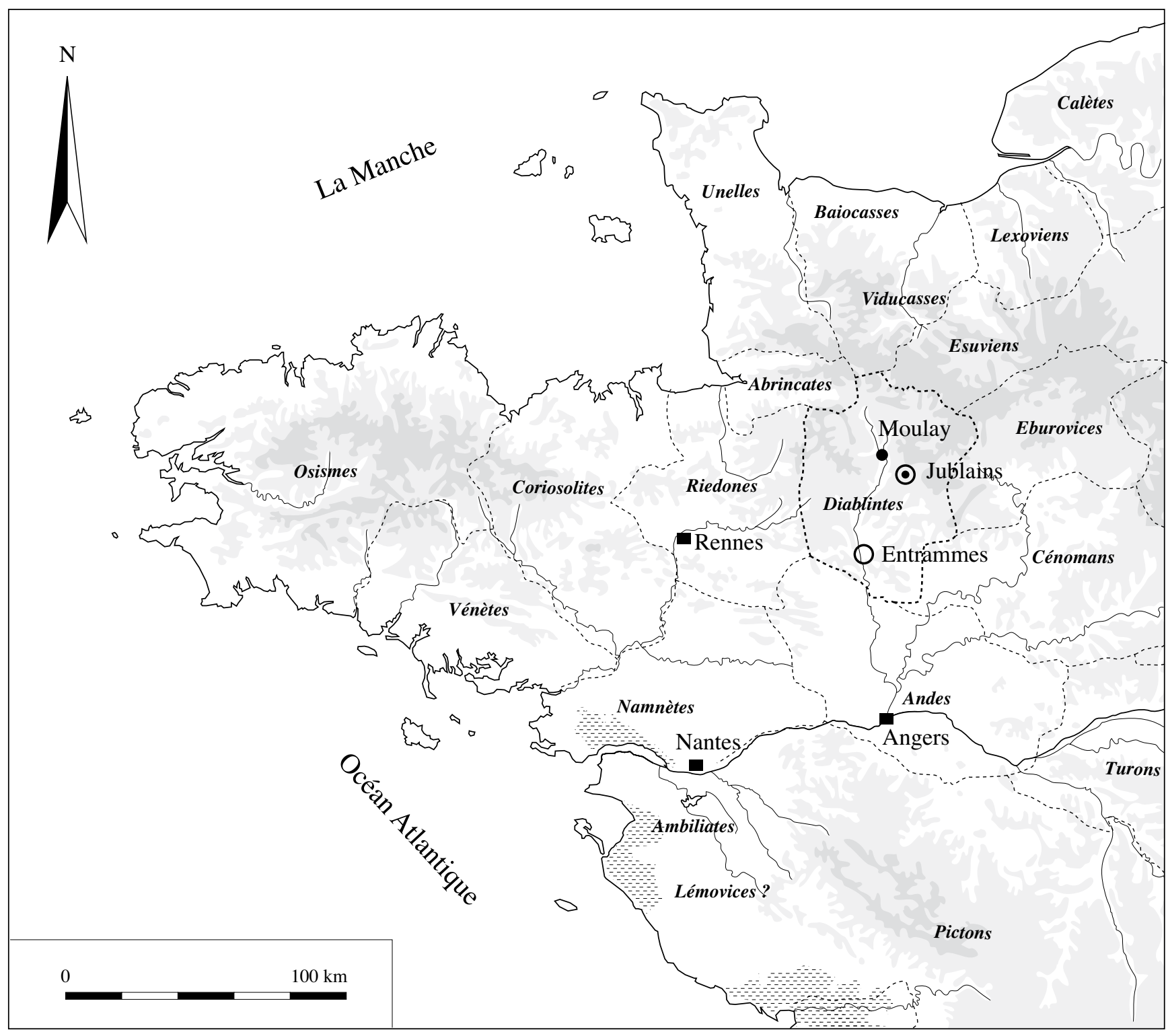

Figure 1 : Jublains - Nouiodunum (Mayenne), chef-lieu de cité des Aulerques Diablintes : carte de localisation (d'après Le Goff, 2003). Figure 1: Jublains - Nouiodunum (Mayenne), capital of the Aulerci Diablinti: location map. 


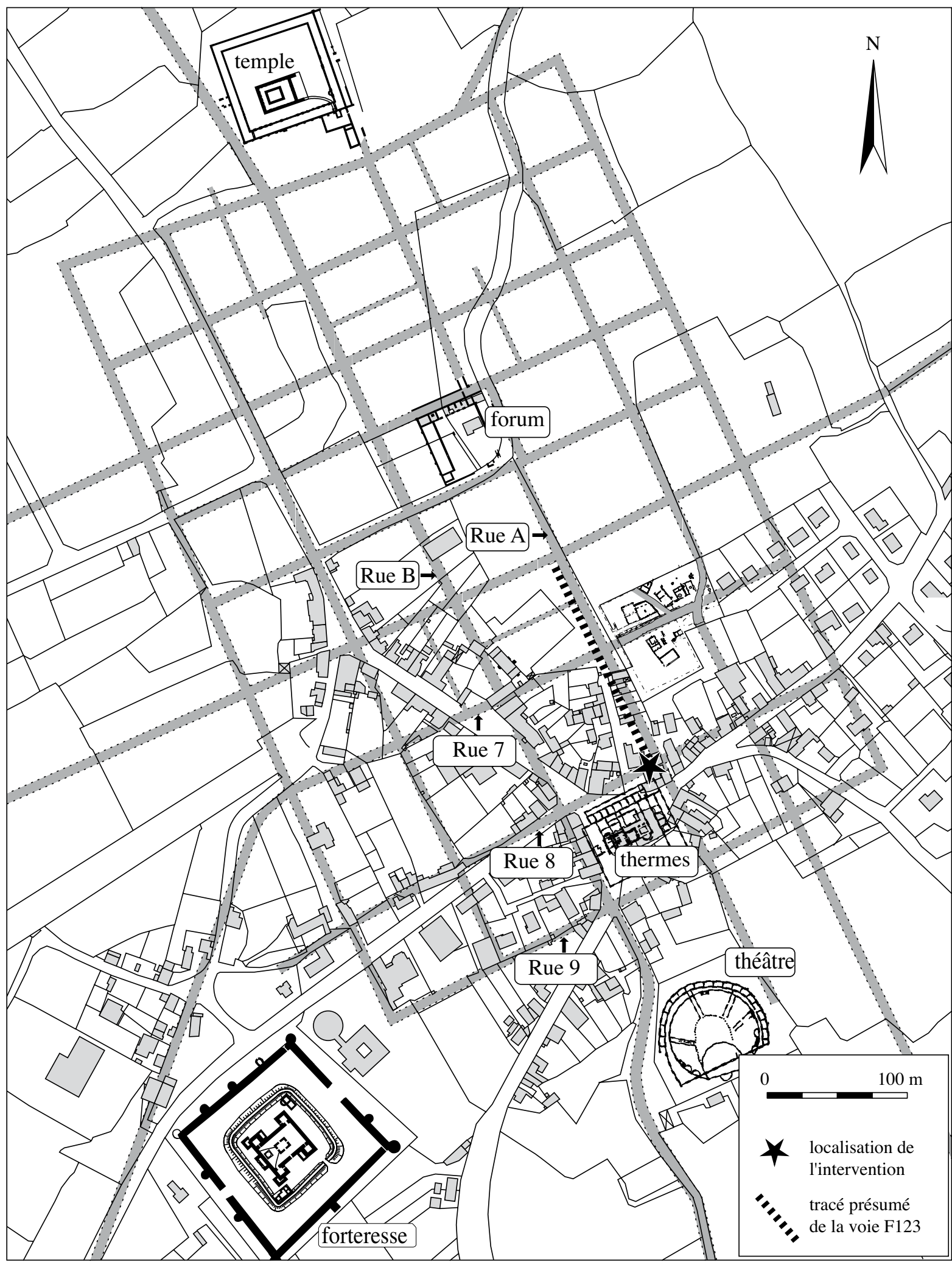

Figure 2 : Jublains (Mayenne) : plan schématique de l'agglomération antique par rapport au bourg actuel et localisation de l'intervention archéologique de "l'impasse Romaine " (d'après Bocquet et al., 2004).

Figure 2: Sketch plan of the Antique town over the modern village and location of the "Impasse Romaine" excavation. 
l'aménagement d'un nouveau bureau postal étaient prévus Impasse Romaine, non loin au nord de l'actuelle église ${ }^{1}$.

\section{L'environnement archéologique}

Lîlot urbain antique (A-B/7-8) où est localisé le site de l'Impasse Romaine (ancienne rue du Presbytère) est implanté au cœur de la ville antique. Il est délimité, à l'est et à l'ouest par les rues $\mathrm{A}$ et $\mathrm{B}$, au nord et au sud par les rues 7 et 8 (fig. 2). Cet îlot est situé dans l'axe, approximativement nord-sud, des principaux monuments urbains que sont le temple, le forum, les thermes et le théâtre. Il se trouve immédiatement au nord de l'îlot des thermes (Naveau [dir.], 1997, p. 77-104). L'îlot $\mathrm{A}-\mathrm{B} / 7-8$ a fourni de nombreuses informations, parfois anciennes mais toujours partielles (ibid., p. 101-102 et fig. 58). De nombreux murs antiques et une salle sur hypocauste ont été découverts, ainsi que des enduits peints, des coquilles d'huîtres et des fragments de céramique (dont des vaisselles sigillées d'Argonne du Bas-Empire). Signalons aussi la découverte, Impasse Romaine, par René Diehl, d'une importante construction de $22 \mathrm{~m}$ de côté, à quelques mètres seulement au nord de la présente fouille (Naveau, 1992, fig. 67, p. 70; Naveau [dir.], 1997, p. 101-102).

\section{Topographie et géologie}

La ville de Jublains est localisée à une altitude d'un peu plus de $150 \mathrm{~m}$, à l'extrémité méridionale d'un vaste plateau granitique primaire au sommet légèrement bombé, qui domine nettement la plaine d'Évron au sud. Le site de l'Impasse Romaine est situé sur la partie haute de ce sommet, à une altitude de $153 \mathrm{~m}$ environ. Son substrat, comme ailleurs à Jublains, est constitué d'une arène granitique compacte, de teinte jaune à beige (Naveau, 1992; Naveau [dir.], 1997; Bocquet et al., 2004).

\section{Les conditions d'intervention}

Une phase préliminaire menée par Jean-Philippe Bouvet, du Service régional de l'Archéologie des Pays-de-la-Loire, a consisté en l'ouverture de six sondages maillant la partie accessible de la parcelle (S1 à S6 : fig. 3). Le même maillage a été conservé à la fouille. Le diagnostic a été mené jusqu’à l'apparition des couches archéologiques et la surface explorée concerne une centaine de mètres carrés environ ${ }^{2}$.

1. Site $\mathrm{n}^{\circ}$ 53.122.145 AH, parcelle A787 du cadastre de 1985. Sa surface est d'environ $190 \mathrm{~m}^{2}$. L'élaboration de cette publication a reçu un soutien financier de l'INRAP.

2. La fouille a été effectuée du $1^{\text {er }}$ juillet au 15 août 1995 sous la responsabilité de G. Guillier (1995a) et sous le contrôle du Service régional de l'Archéologie des Pays-de-la-Loire.
Liée à l'étude du mobilier archéologique, l'approche stratigraphique du site a permis, pour la période antique, de définir quatre phases principales d'occupation (dénommées I à IV). Remarquons l'absence de vestiges laténiens sur la parcelle fouillée, alors qu'ils sont attestés sur le site de la Grande-Boissière, à seulement $100 \mathrm{~m}$ au nord (Bocquet $e t$ al., 2004, p. 134-135). L'exiguïté de la parcelle explorée peut en être la cause; il est aussi envisageable que l'extension de l'agglomération gallo-romaine soit bien plus importante que celle de l'occupation gauloise.

\section{LES PREMIÈRES TRACES DE L'OCCUPATION : UNE VOIE PRÉ-FLAVIENNE}

\section{La voirie}

Cette " phase I " caractérise les traces archéologiques les plus anciennes. Il s'agit d'une vaste structure fossoyée (F123), creusée dans l'arène granitique, orientée approximativement nord-sud et présentant un fond relativement plat (cote $152,70 \mathrm{~m})^{3}$, ainsi que de sa première phase de comblement matérialisée par la couche 1048 (fig. 3; fig. 4, coupe 1). Cette fosse borde au sud la future rue 8 où elle se caractérise par une rapide remontée du fond de la structure. L'emprise de la fouille n'a pas permis, en revanche, de déterminer la limite nord de F123. Toutefois, les recherches effectuées par Jacques Naveau sur l'îlot $A-B / 5-7$, situé immédiatement au nord, mentionnent la présence d'une vaste dépression à fond plat, large de $7 \mathrm{~m}$ environ et reconnue sur environ $200 \mathrm{~m}$ (Naveau, 1992, fig. 28 p. 47; Naveau [dir.], 1997, p. 77-79), dépression qui se situerait dans l'axe de F123 et en constituerait, de la sorte, le prolongement.

Cette vaste structure, parallèle à la rue A conservée (fig. 2), demeure difficile à interpréter. S'agit-il d'un premier état de cette voie comme l'estime Jacques Naveau (1997, p. 77-79)? Un certain nombre d'indices permettent de privilégier cette hypothèse. Les premiers ont trait au tracé de cette dépression F123. D'après les observations de Jacques Naveau (1992, fig. 28 p. 47 ; 1997, p. 77-79), elle est parallèle, à l'ouest, à la voie $\mathrm{A}$ le long de laquelle elle a été observée en différents points sur plus de $250 \mathrm{~m}$ (fig. 2). Ensuite, ce vaste creusement peut s'expliquer par la nécessité d'extraire les matériaux nécessaires pour justement construire la nouvelle voie $A$, plus à l'est. Enfin, le mobilier présent sur le fond de la structure est nettement pré-flavien, période à la fin de laquelle est justement aménagée la trame viaire orthogonale (Naveau, 1986).

3. Les cotes d'altitudes sont données en m NGF (nivellement général de la France). 
Figure 3 : Jublains, "Impasse Romaine " : implantation des secteurs de fouille $S 1$ à $S 6$, des coupes stratigraphiques et extension de la structure fossoyée F123 (phase I).

Figure 3: Location of S1-S6 excavation sectors and of stratigraphic sections, and extension of dug structure F123 (phase I).

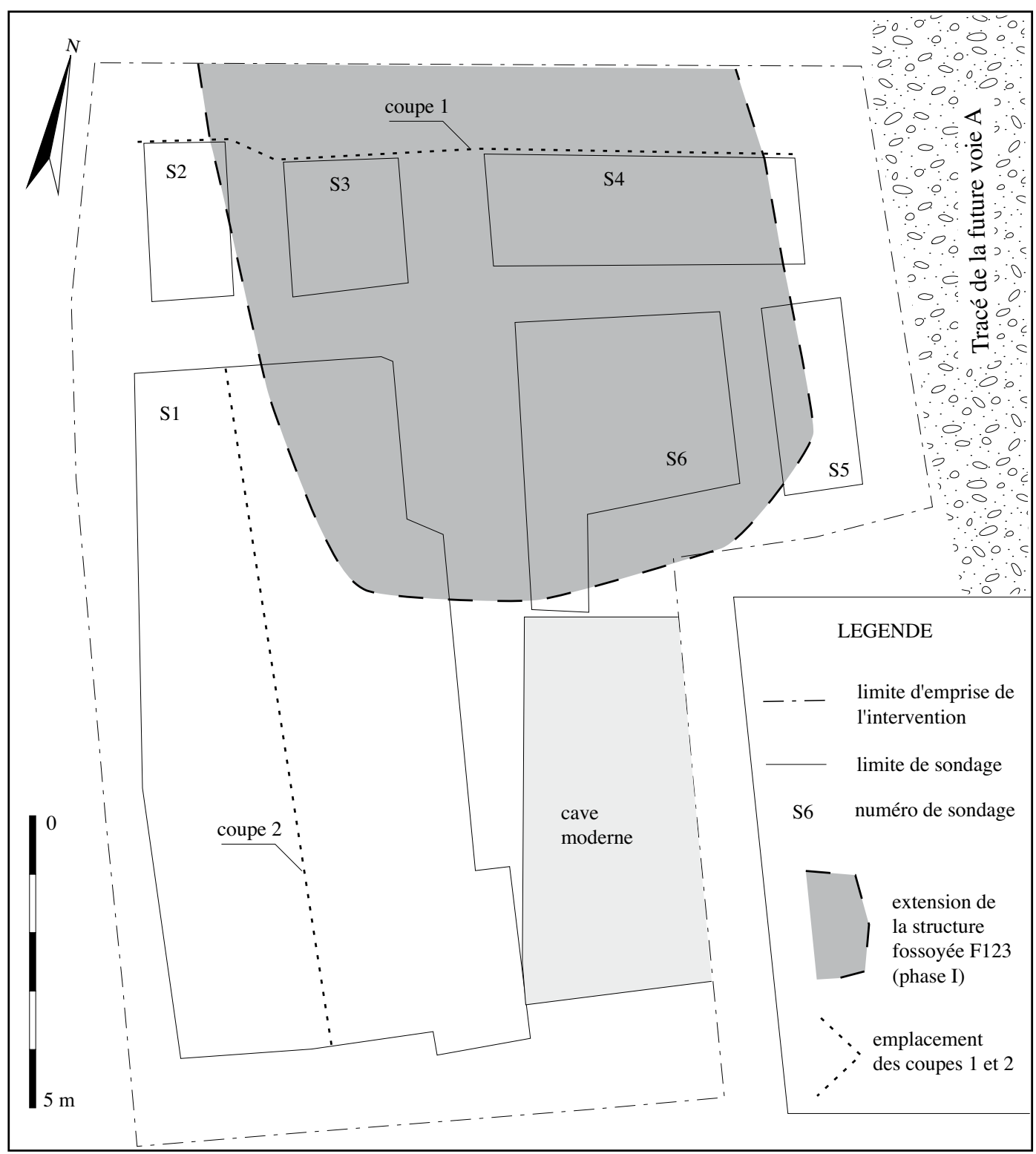

\section{Le mobilier archéologique}

Le tableau 1 présente, de manière simplifiée, le mobilier céramique attribuable à cette phase I.

La céramique sigillée comporte six vases tibéro-claudiens, essentiellement des assiettes (Ritt. 1, Drag. 18, Drag. 15/17) et une coupelle (Drag. 24/25) issues des ateliers de MillauLa Graufesenque, complétées de deux pièces Drag. 29 de Montans et de Lezoux. Les sigillées des décennies suivantes, mais toujours antérieures à la période flavienne, sont également dominées par les vases de Millau-La Graufesenque (8 pièces sur 9), avec des assiettes de séries typologiques identiques (Drag. 18 et Drag. 15/17), complétées d'un corpus de coupelles comportant notamment trois formes bilobées

\begin{tabular}{|c|c|c|c|c|c|c|c|c|c|}
\hline & sigillée & PF & fumigée & CCC & CCS & amph. & mort. & mod. & totaux \\
\hline nombre & 61 & 0 & 66 & 271 & 45 & 18 & 2 & 15 & 478 \\
\hline$\%$ & 12,8 & 0 & 13,8 & 56,7 & 9,4 & 3,8 & 0,4 & 3,1 & 100 \\
\hline
\end{tabular}

Tableau 1 : Jublains, "Impasse Romaine " : comptage récapitulatif du mobilier céramique de la phase I, en nombre de tessons et en pourcentages ( $\mathrm{PF}$ : parois fines; $\mathrm{CCC}$ : céramique commune claire; CCS : céramique commune sombre; amph. : amphores; mort. : mortiers; mod. : céramique modelée).

Table 1: Summary of Phase I ceramics assemblage, in number of shards and percents (PF: thin walled; CCC: common clear; CCS: common dark; amph: amphorae; mort: mortars; mod: modelled ware). 
Drag. 27 (fig. 5, $\mathrm{n}^{\circ} 1$ ), ainsi que deux fragments de coupes carénées moulées Drag. 29 (dont le $\mathrm{n}^{\circ} 2$ ). Une dernière pièce est issue des ateliers de Lezoux (assiette Drag. 15/17).

La vaisselle de table comporte également des céramiques fumigées : une coupe Menez 96 (fig. 5, n 3), un vase bobine de forme Menez 125C $\left(\mathrm{n}^{\circ} 4\right)$ et une petite coupe à panse arrondie $\left(n^{\circ} 5\right)$. Les cruches sont représentées par des exemplaires à lèvre en bourrelet ( $n^{\circ} 6$ et 7$)$, tandis qu'un petit vase à lèvre moulurée $\left(n^{\circ} 8\right)$ comporte un décor de peinture ocrée. Est également attesté dans ce lot un grand vase culinaire en céramique commune grise $\left(\mathrm{n}^{\circ} 9\right)$. Les premières années de l'occupation du site ont aussi livré un petit lot de céramique modelée; nous y relevons une coupe ou écuelle $\left(\mathrm{n}^{\circ} 10\right)$, une assiette $\left(\mathrm{n}^{\circ} 11\right)$ ainsi qu'un fragment de panse à décor côtelé $\left(n^{\circ} 12\right)$. Ces éléments modelés, à pâte brune et aux surfaces soigneusement lissées, se retrouvent, mais en faible nombre, dans les contextes archéologiques de Jublains (Chuniaud et Mortreau, 2002, p. 174), ainsi que chez leurs voisins cénomans (Guillier, 1995b) où les formes sont relativement proches. Le tesson $\mathrm{n}^{\circ} 12$, au décor côtelé, présente des parallèles à Jublains même, sur la nécropole méridionale (Boissel et Diehl, 1972, pl. IV) avec un gobelet imité de la forme en verre Isings 67. D'autres éléments comparables à décor côtelé sont relevés au Mans (Guillier, 1995b, p. 218-219.)

Enfin, cet ensemble comporte des fragments d'amphores Dressel 20 (fig. $5, \mathrm{n}^{\circ} 13$ ), conteneur à huile de Bétique d'époque claudienne, mais aussi une amphore Dressel 7/11 à pâte sableuse, contemporaine et probablement d'origine lyonnaise $\left(\mathrm{n}^{\circ} 14\right)$. Notons aussi la présence d'un col de Pascual 1 à la pâte blanc-rose $\left(\mathrm{n}^{\circ} 15\right)$, d'origine ibérique et attribuable à l'époque augusto-tibérienne.

\section{L'AMÉNAGEMENT DE LA VOIE DÉCUMANE 8}

\section{Les structures}

La phase II du site est matérialisée par l'aménagement d'une voie, de niveaux de circulation, de caniveaux et de murs (fig. 6).

L'axe nouvellement créé correspond à la voie décumane 8 , qui longe au nord les thermes antiques (fig. 2). Cette voie, large de $7 \mathrm{~m}$ environ, comporte plusieurs niveaux de construction constitués de blocs de granite enrobés par de l'arène granitique fortement damée (fig. 4, coupe 2 : F1062, F1039 et F1061), à l'exception de F1040, constitué de blocs de granite enrobés par des scories de fer. Des alignements de gros blocs de pierres forment une bordure aux différents remblais de rue. Ce type d'aménagement a déjà été reconnu à Jublains, notamment le long du cardo B (Naveau, 1997, p. 84), mais aussi au Mans (Sarthe), par exemple sur le site de la Cité-Judiciaire
(Bouvet, 2001, p. 320). Parallèlement à la voie 8 , au nord de celle-ci, un petit mur-bahut orienté est-ouest est installé (F1109), ainsi que son retour nord-sud (F1025/1110). Ces deux murs, formant un angle à peu près droit, limitent au nord-ouest une succession de remblais appartenant soit à une construction bordant immédiatement la voie 8 , soit plus vraisemblablement à un trottoir courant le long de celle-ci.

Par ailleurs, des dépressions formant égouts se sont succédées au nord de la voie 8 (F1065 et 1066), tandis qu'au sud différents remblais (F1111 et 1035) ont été apportés, signalant la présence d'un trottoir au nord de la voie 8 . Ces derniers remblais sont recouverts par une couche de destruction (celle des thermes?) : F1034.

\section{Quelques éléments de chronologie}

Des monnaies julio-claudiennes, flaviennes et du début de la période antonine sont disséminées dans les égouts et les remblais de bord de voie ${ }^{4}$. La monnaie M8, émise sous le règne de Néron en l'an 64 et mise au jour dans une fine couche (F1063 : fig. 4, coupe 2), immédiatement sous le premier niveau de voie (F1062), constitue un marqueur chronologique important. Elle vient, en effet, confirmer l'hypothèse d'une mise en place de la trame viaire à la période flavienne émise par Jacques Naveau (1992, p. 44), hypothèse renforcée par ailleurs à Jublains par les découvertes effectuées sur les îlots de la Grande-Boissière où une restructuration du quartier a pu être observée à cette époque (Bocquet et al., 2004, p. 139 et suiv.).

\section{LE MUR NORD DES THERMES ANTIQUES ET LES REMBLAIS}

\section{Les structures de la phase III et leur chronologie}

La phase III est matérialisée par l'aménagement du mur F1070, situé en limite sud de la parcelle fouillée (fig. 4, coupe 2 ; fig. 6 , sondage $S 1$ ), ainsi que par l'apport de différents remblais matérialisant la fin du comblement de la vaste dépression F123 dont le creusement et les premiers remblais témoignaient de la phase I. Stratigraphiquement, le mur nord F1070 semble recouper une séquence importante de remblais situés au nord de la rue $8:$ le trottoir F1111 et 1035 (fig. 4, coupe 2). La maçonnerie a été mise au jour sur $5 \mathrm{~m}$ de longueur d'est en ouest et sur $0,90 \mathrm{~m}$ de largeur. Le mur se présente sous la forme d'une mince couche de mortier englobant quelques blocs de granite, puis d'un radier

4. Ces monnaies sont localisées stratigraphiquement par un astérisque sur les coupes présentées (fig. 4); leur description est donnée infra (annexe par P.-A. Besombes). 


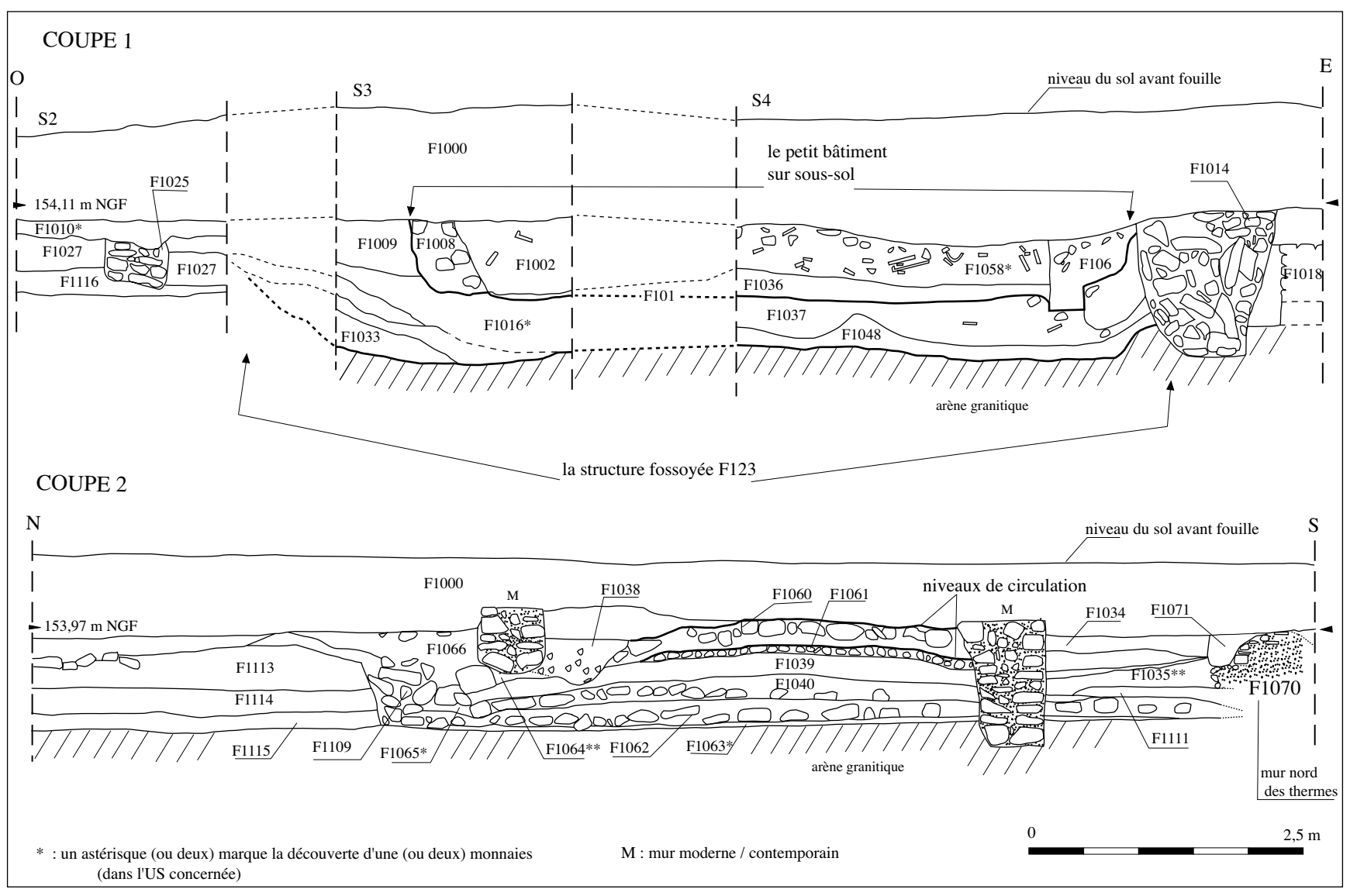

Figure 4 : Jublains, "Impasse Romaine ", coupe 1 : la structure fossoyée F123 (phase I) et le petit bâtiment sur sous-sol (Phase IV). Coupe $2:$ la voie 8 et le mur nord des thermes, F1070.

Figure 4: Section 1 : dug structure F123 (Phase I) and small building with cellar (Phase IV). Section 2: street 8 and nothern wall of thermae.

de fondation, large de $0,80 \mathrm{~m}$, formé d'un mortier maigre ennoyant de petits blocs de granite de 0,05 à $0,10 \mathrm{~m}$ de côté. Ces fondations sont surmontées, en retrait de $0,15 \mathrm{~m}$, par un unique rang de moellons lui-même placé sous une assise de briques, ultime vestige visible de l'élévation du mur.

Le mur F1070 se situe au sud de la rue 8 et appartient donc à l'îlot $\mathrm{A}-\mathrm{B} / 8-9$, celui des thermes (Naveau [dir.], 1997 , p. $89-91$ et $102-103)$. Il constitue, selon toute vraisemblance, un élément de l'angle nord-est de la galerie double, qui ceignait au nord et à l'est l'édifice thermal antique localisé sous l'église actuelle, hypothèse reprise dans la dernière synthèse sur Jublains (ibid., fig. 63).

D'un point de vue chronologique, les seuls éléments sur le site permettant d'avancer une datation sont la présence de deux monnaies, de Claude et de Néron, dans les remblais traversés par le mur F1070 (cf. Annexe, M4 et M9). En revanche, les thermes ont été datés de manière plus précise, de la fin des Flaviens ou du début des Antonins d'après leur plan linéaire classique (Naveau, 1992; Naveau [dir.], 1997, p. 91).
Quant à la couche F1034 (fig. 4, coupe 2), dont la constitution ne peut être située dans le temps, elle semble caractériser un épisode de destruction, vraisemblablement celui des thermes. Elle recelait des pavements de calcaire et de marbre, tandis que F1071 (fig. 4, coupe 2) correspond à une phase de récupération des matériaux du mur nord de ces mêmes thermes.

\section{Présentation du mobilier archéologique}

Le tableau 2 présente, de manière simplifiée, le mobilier céramique attribuable à la phase III, essentiellement issu des niveaux supérieurs du comblement de F123 et qui se compose d'un riche corpus de céramique sigillée. Celui de la période flavienne comporte onze vases. Huit sont liés aux productions de Millau-La Graufesenque avec, pour l'essentiel, des formes du service Ve. A et des assiettes Drag. 18. Trois vases de Lezoux complètent cette série avec deux coupes moulées Drag. 37 et une coupelle Drag. 27. Pour les décennies suivantes, jusqu'au milieu du $\mathrm{II}^{\mathrm{e}}$ siècle, le corpus se compose majoritairement de pièces du centre de la Gaule, 


\begin{tabular}{|c|c|c|c|c|c|c|c|c|c|}
\hline & sigillée & PF & fumigée & CCC & CCS & amph. & mort. & mod. & totaux \\
\hline nombre & 59 & 4 & 35 & 251 & 75 & 16 & 0 & 14 & 454 \\
\hline$\%$ & 13 & 1 & 7,7 & 55,3 & 16,5 & 3,5 & 0 & 3,1 & 100 \\
\hline
\end{tabular}

Tableau 2 : Jublains, «Impasse Romaine » : comptage récapitulatif du mobilier céramique de la phase III, en nombre de tessons et en pourcentages (PF : parois fines; CCC : céramique commune claire; CCS : céramique commune sombre ; amph. : amphores; mort. : mortiers; mod. : céramique modelée).

Table 2: Summary of Phase III ceramics assemblage (legend as for Tabl. 1). issues de Lezoux mais aussi d'autres centres de production de la vallée de l'Allier (11 sur 13), complété de deux vases $\mathrm{du}$ Centre-Ouest. Le répertoire est alors très varié : coupelles Lez. 14 et 24, Drag. 27 (fig. $7, \mathrm{n}^{\circ}$ 1), assiettes Lez. 15 ( $\mathrm{n}^{\circ}$ 2), Lez. 25, 32, 45 et 55, coupes Drag. 30 et 37 . Les deux vases du Centre-Ouest correspondent à une coupe cylindrique moulée Drag. 30 et à un mortier Curle 21. Quelques vases de la seconde moitié du II $^{\text {e }}$ siècle (six individus) figurent également parmi ce lot. Il s'agit pour l'essentiel de sigillées du centre de la Gaule, correspondant à des vases moulés (Drag. 37) ou à des mortiers (Curle 21).

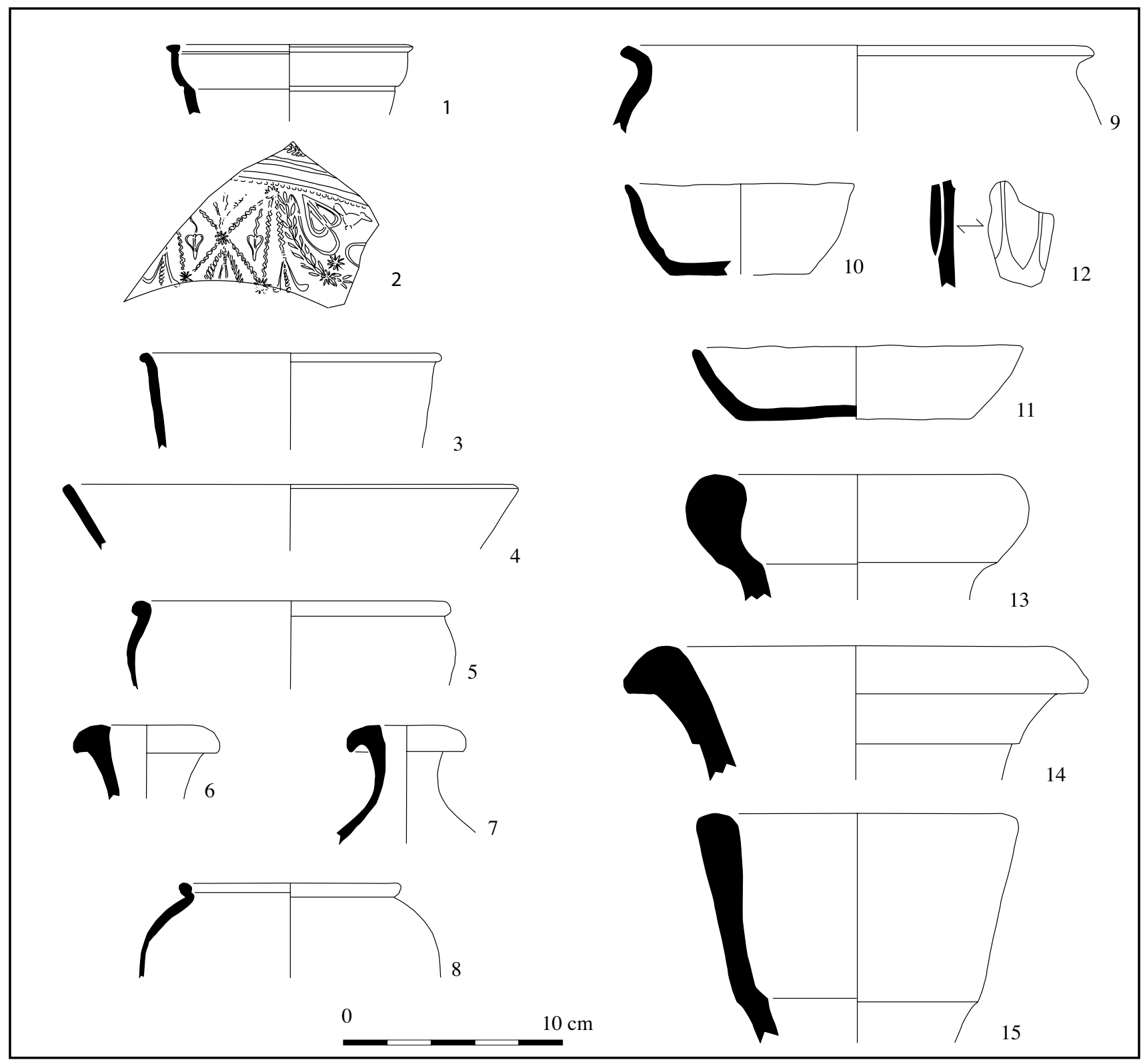

Figure 5 : Jublains, «Impasse Romaine » : le mobilier de la phase I, julio-claudien.

Figure 5: Julio-Claudian Phase I assemblage. 
Figure 6 : Jublains, "Impasse

Romaine " : la rue 8 (phase II), le mur nord des thermes F1070 (phase III) et le petit bâtiment sur sous-sol (phase IV). Figure 6: Street 8 (Phase I), F1070 northern wall of thermae (Phase III) and the small building with cellar(Phase IV).

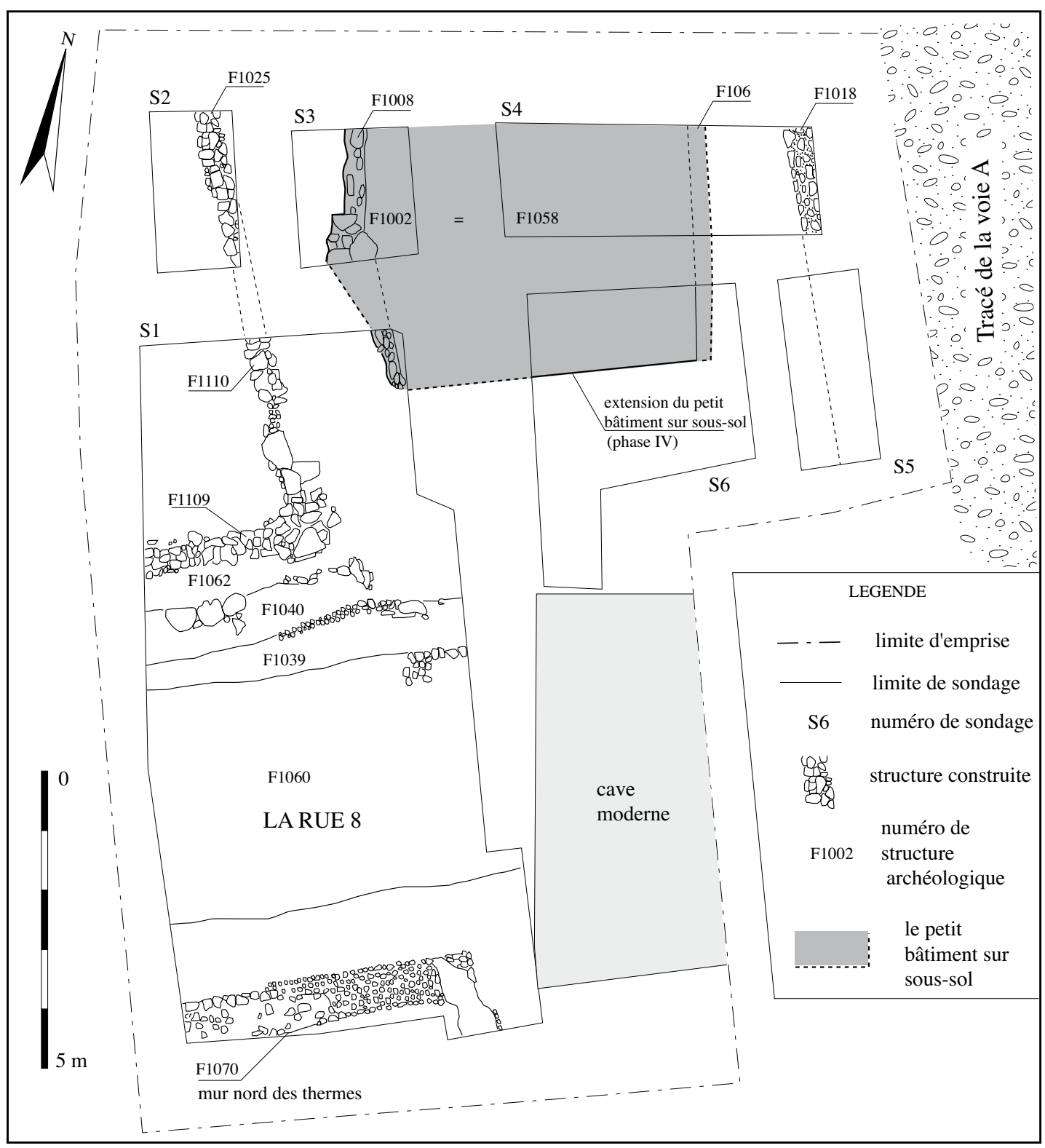

portants remblais, peut-être plusieurs fois remaniés, venant clore cette grande structure fossoyée.

Nous avons aussi relevé la présence de "palets " taillés dans des tuiles (fig. $7, \mathrm{n}^{\circ} 15$ ) ou dans des tessons d'amphores $\left(\mathrm{n}^{\circ} 16\right)$ et de " jetons " découpés dans des tessons de panses de cruche et aux tranches soigneusement lissées ( $n^{\circ} 17$ à 19); l'un d'eux est percé en son centre et décoré de sept incisions rayonnantes $\left(\mathrm{n}^{\circ} 20\right)$.

\section{LE PETIT BÂTIMENT SUR SOUS-SOL ET SON DODÉCAÈDRE}

Aménagé dans les remblais supérieurs de F123 (phase III), un petit bâtiment sur sous-sol excavé, caractérisant la pha- 

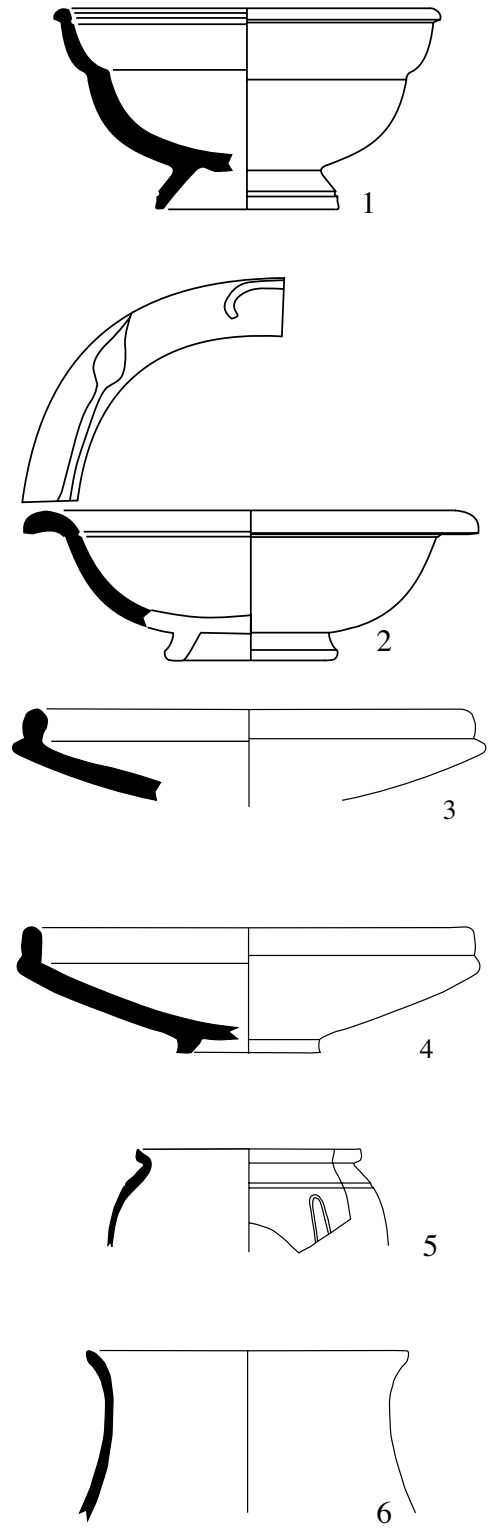
6
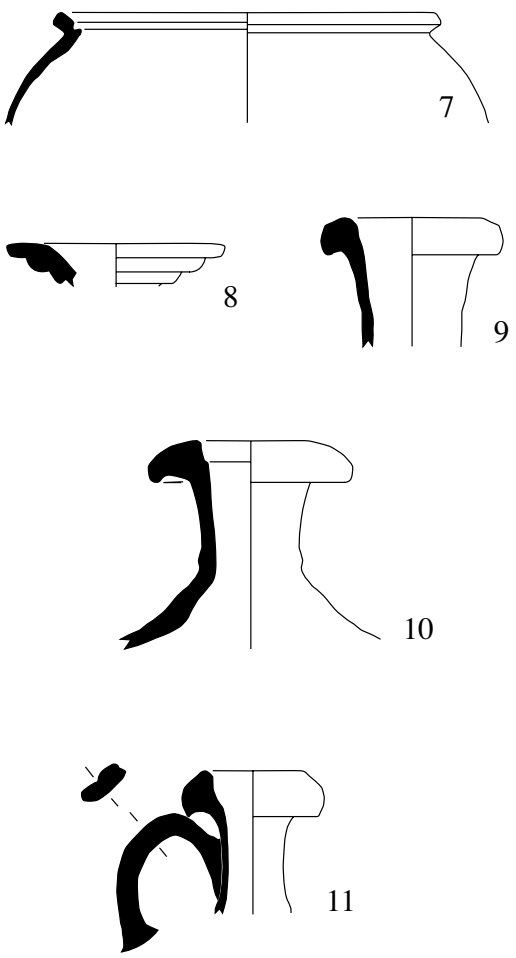

11

0
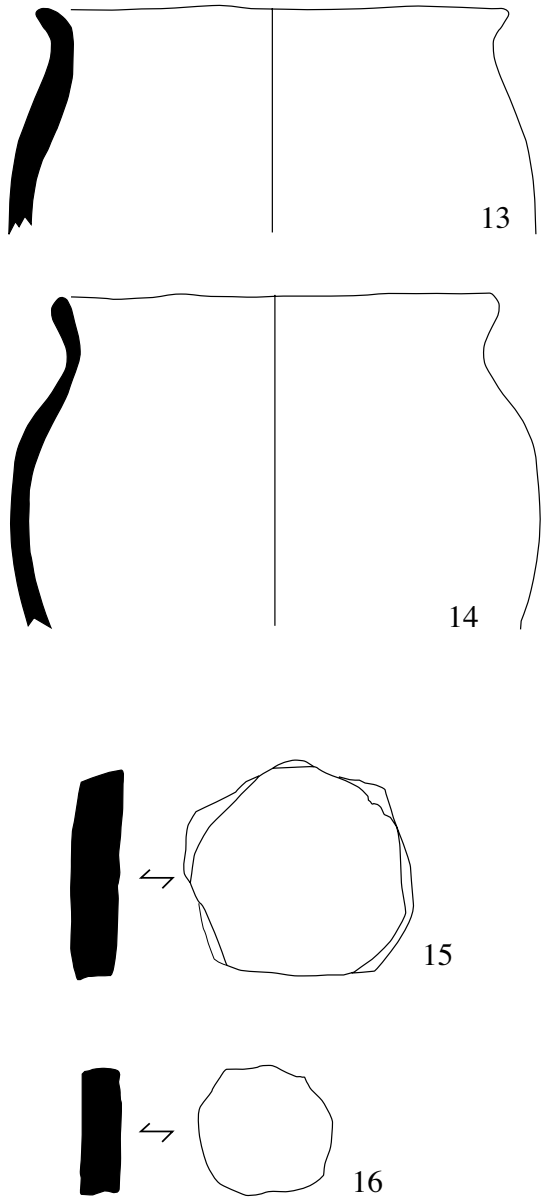

16

5
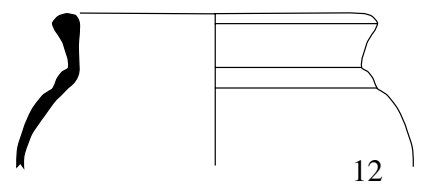

17
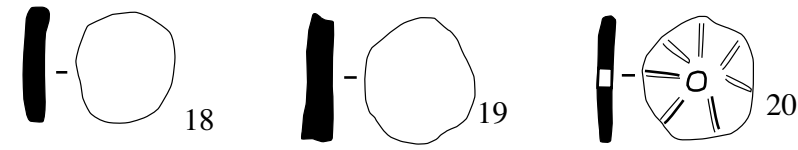

$$
0
$$
(1)

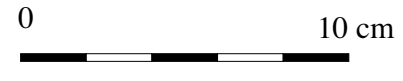

Figure 7 : Jublains, "Impasse Romaine » : le mobilier de la phase III, Flaviens et début Antonins.

Figure 7: Flavian - early Antonians Phase III assemblage.

se IV, et dont seule la partie méridionale a été fouillée, est situé dans la partie nord de l'emprise de fouille (fig. 6). Il a pu être reconnu au sein des sondages $S 1, S 3$, S4 et $S 6$; trois séquences - aménagement, occupation et destruction - y ont été distinguées. Quelques autres couches peuvent être rattachées à la phase IV : ce sont le dernier niveau de la voie (F1060), ainsi que le segment de "fossé-égout » qui lui est lié et qui court sur sa bordure nord : F1038 (fig. 4, coupe 2).

\section{L'aménagement du bâtiment sur sous-sol}

Sa mise en place a nécessité le creusement d'une excavation au sein des remblais de comblement de F123 (phase III). Sa largeur selon un axe est-ouest est de 6,30 m (fig. 4, coupe 1) mais elle n'a été reconnue, selon un axe nord-sud, que sur 4,50 m (fig. 6). Il est possible d'estimer la profondeur de l'excavation à $1 \mathrm{~m}$ environ par rapport à la cote supérieure des remblais. 
Le mode de construction des parois n'a été réellement mis en évidence que sur son côté ouest. Il s'agit d'un petit solin construit à l'aide de blocs de granite assemblés sans mortier, directement contre la paroi de l'excavation (fig. 4, coupe 1, F1008). La base du solin se situait au même niveau que le fond de l'excavation. Les matériaux constitutifs des murs placés à l'est et au sud ayant été récupérés (F106), leur nature nous est inconnue; il est toutefois vraisemblable qu'ils devaient être similaires à ceux du solin ouest. Notons aussi que les orientations relevées sur cette structure se placent dans la filiation de celle de la structure F123 de la phase I (fig. 3) mais aussi des vestiges des phases II et III (fig. 6).

\section{Occupation et destruction}

L'occupation du sous-sol de ce bâtiment est matérialisée par une fine couche de sable de teinte verte, meuble, qui en tapissait le fond (fig. 4, coupe $1:$ F1036) sur une épaisseur de 0,05 à $0,20 \mathrm{~m}$. Elle est surmontée d'une épaisse couche (F1002=1058), constituée d'un mélange de terre brune, de charbon de bois, de torchis rubéfié (avec de très nettes traces de clayonnage), ainsi que de nombreux fragments de tegulae et imbrices. Ces matériaux sont très probablement issus de la destruction par incendie du bâtiment; les solins le limitant devaient être surmontés d'une structure en clayonnage et le toit couvert de tuiles. Ce mode de construction est assez commun dans l'ouest de la Gaule, aussi bien en milieu rural qu'urbain. À la période julio-claudienne, certains bâtiments sont entièrement en bois, y compris la partie excavée. Par la suite, la plupart d'entre eux présentent un soubassement de pierres liées au mortier, comme nous en avons des exemples au Mans (Bouvet, 2001). Le bâtiment de Jublains se singularise localement par le fait qu'il soit excavé d'environ $1 \mathrm{~m}$, traduisant la présence d'un vide sanitaire, sinon d'une cave plus ou moins profondément creusée.

\section{Éléments de chronologie}

Le mobilier associé à cette phase IV est essentiellement constitué de céramique ${ }^{5}$ (tableau 3 ); les autres vestiges, métalliques, sont présentés infra.

La céramique sigillée avec une représentation d'environ $15 \%$ des fragments (NMI de 30 vases), constitue l'essentiel de la vaisselle de table de la phase IV (tableau 4 ; fig. 8). Si le corpus se compose d'une quinzaine de formes, seules, en fait, quatre d'entre elles dominent le répertoire, avec pas moins de 60 à $72 \%$ des exemplaires suivant le mode de comptage. Il est intéressant de noter également que la plu-

5. Est également présente une monnaie résiduelle d’Hadrien (M13) $-c f$. annexe.

\begin{tabular}{|l|r|r|r|r|r|r|r|r|}
\hline & Sigillée & mét. & amph. & mort. & CCC & CCS & mod. & totaux \\
\hline Phase IV & 80 & 19 & 12 & 10 & 302 & 87 & 13 & 522 \\
\hline$\%$ & 15,1 & 3,6 & 2,3 & 1,9 & 57,8 & 16,7 & 2,5 & 100 \\
\hline
\end{tabular}

Tableau 3 : Jublains, «Impasse Romaine » : comptage récapitulatif du mobilier céramique de la phase IV, en nombre de tessons et en pourcentages (mét : céramique métallescente; amph. : amphores ; mort. : mortiers; CCC : céramique commune claire; CCS : céramique commune sombre; mod. : céramique modelée).

Tableau 3: Summary of Phase IV ceramics assemblage (mét : metallicware; the rest as for Tabl. 1).

part de ces vases présentent les caractéristiques techniques, morphologiques et/ou stylistiques propres aux productions du $\mathrm{III}^{\mathrm{e}}$ siècle des ateliers du centre de la Gaule, majoritairement ceux de Lezoux.

Leur répartition au sein des différentes unités stratigraphiques du bâtiment et de son environnement montre clairement que ce bâtiment a été utilisé et détruit au cours de ce siècle et plus vraisemblablement lors de sa première moitié. En témoigne notamment la présence d'une coupe Drag. 37 et d'une assiette L043/C23, typique de cette période et qui caractérise le mieux la séquence d'occupation du bâtiment.

Les décors sur vases moulés peuvent être rattachés à trois styles décoratifs. Celui de MARCvs est le plus récent puisque l'officine est en activité à partir de 210 et que certains des moules sont encore utilisés après la disparition de celle-ci au cours de la seconde moitié du III ${ }^{\mathrm{e}}$ siècle (fig. 8, $\mathrm{n}^{\circ} 1$ ). Aujourd'hui, de nombreux assemblages de référence valident ces propositions chronologiques : au Mans (Sarthe) ${ }^{6}$, à Corseul (Côtes-d'Armor) ${ }^{7}$, à Vieux (Calvados) ${ }^{8}$, aux Murgets (Loiret) ${ }^{9}$, à Santeuil (Val-d'Oise) ${ }^{10}$, etc.

Les autres styles décoratifs attestés sont ceux de PATERNVs II et PRISCVS/CLEMENS, dont l'activité principale de création de décors peut être située dans le courant de la seconde moitié $\mathrm{du} \mathrm{II} \mathrm{I}^{\mathrm{e}}$ siècle, mais dont, là encore, les moules conservent une longue durée de vie. Tel est tout particulièrement le cas pour le second, puisque les caractéristiques techniques et morphologiques du vase-support (fig. $8, \mathrm{n}^{\circ} 2$ ) incitent à situer sa fabrication au cours du III $^{\mathrm{e}}$ siècle ${ }^{11}$.

6. Fouille de l'îlot 7, puit F1, datation fin du III ${ }^{\mathrm{e}}$ siècle : Delage et Guillier, 1997.

7. Fouille de Monterfil II, Horizon IV, datation du début du III ${ }^{\mathrm{e}}$ siècle : Ferrette, 2003.

8. Fouille du Bâtiment A-1173, remblai de la deuxième moitié du III $^{\mathrm{e}}$ siècle : Jardel, 2002.

9. Dépotoir de la première moitié du III ${ }^{\mathrm{e}}$ siècle : Moireau, 1992.

10. Phase 4, remblai de la fin du III ${ }^{\mathrm{e}}$ siècle : Philippe et al., 1996.

11. La présence, au sein des niveaux du $\mathrm{III}^{\mathrm{e}}$ siècle, de productions comportant un décalage chronologique entre fabrication du vase et du décor est très fréquente. Un vase de PRISCVs/CLEMENs tardif $a$, par exemple, été retrouvé à Hermesnil (Seine-Maritime) dans un contexte de la première moitié du III ${ }^{\mathrm{e}}$ siècle (Coffineau et Dubant, 2002). 


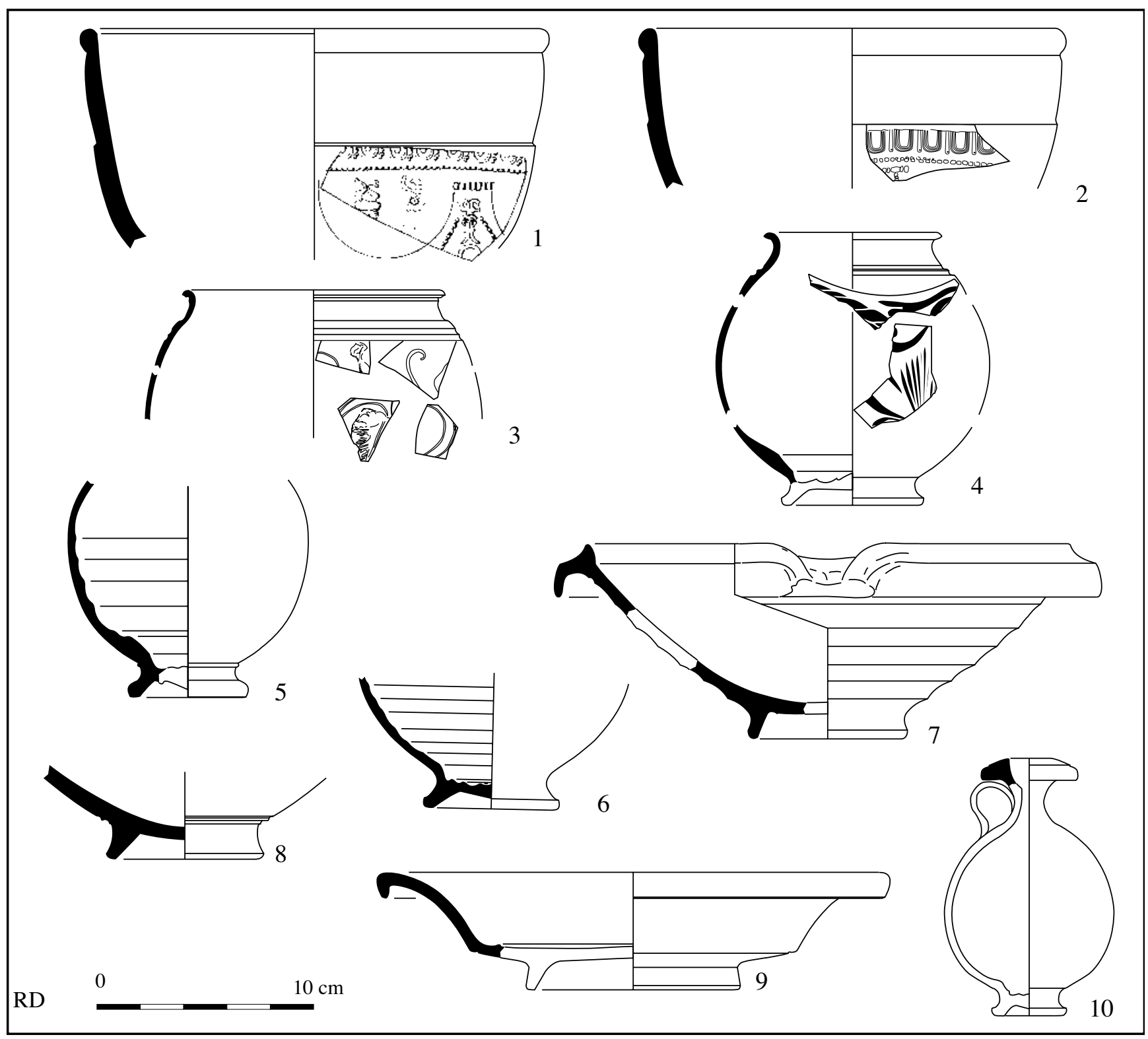

Figure 8 : Jublains, «Impasse Romaine » : le mobilier de la phase IV : la céramique sigillée.

Figure 8: Assemblage of Phase IV: terra sigillata.

Les formes ovoïdes L105/D72 constituent la deuxième série la plus abondante des sigillées de ce corpus car pas moins de sept vases sont attestés (fig. $8, n^{\circ} 3$ à 6 ). Leurs parois comportent des décors en relief (applique et barbotine, $\left.n^{\circ} 3\right)$ ou des décors excisés de motifs floraux $\left(n^{\circ} 4\right)$, caractéristiques des productions lézoviennes du $\mathrm{III}^{\mathrm{e}}$ siècle.

Le mortier Lez. 97 (fig. 8, $\mathrm{n}^{\circ} 7$ et 8 ) et sa variante Lez. 96, ainsi que les assiettes/plats L043/C23 dans leurs évolutions tardives à profil très caréné et lèvre pendante $\left(n^{\circ} 9\right)$ complètent ces lots de vaisselles tardives. Notons enfin la présence d'une bouteille L105 ( $\left.\mathrm{n}^{\circ} 10\right)$, forme plu- tôt rare fabriquée au cours de la seconde moitié du II $^{\mathrm{e}}$ siècle et au $\mathrm{III}^{\mathrm{e}}$ siècle.

Les autres vaisselles recensées (tableau 4), présentes pour chaque forme en un seul exemplaire, correspondent essentiellement à des pièces résiduelles. Seule fait exception une assiette Lez. 55 fabriquée au cours de la seconde moitié du $\mathrm{II}^{\mathrm{e}}$ siècle et dont l'usage lors des décennies suivantes est tout à fait envisageable.

La céramique de cuisine et de stockage est essentiellement représentée par des vases en céramique commune claire, dont le répertoire se compose majoritairement de cruches, notam- 


\begin{tabular}{|c|c|c|c|c|c|}
\hline Prov & Réf typo & & NR & bords & NMIp \\
\hline CG & D37 & & 16 & 8 & 13 \\
\hline CG & L105 (D72) & & 21 & & 12 \\
\hline CG & L097 (C21) & & 6 & & 3 \\
\hline CG & L043 (C23) & $\pi$ & 5 & 3 & 3 \\
\hline CG & L015 (VeA2) & & 1 & 0 & 1 \\
\hline CG & L016 & $F$ & 1 & 1 & 1 \\
\hline CG & L042 & $r$ & 1 & 1 & 1 \\
\hline CG & L044 & & 1 & 1 & 1 \\
\hline CG & L055 & & 1 & 1 & 1 \\
\hline CG & L088 (D38) & & 1 & 1 & 1 \\
\hline & L096 & & 1 & 0 & 1 \\
\hline CG & L105 & & 1 & 1 & 1 \\
\hline CG & ind. & & 19 & 0 & 0 \\
\hline COG & D30 & & 1 & 0 & 1 \\
\hline SG & D18 & r & 3 & 3 & 3 \\
\hline SG & ind. & & 1 & 0 & 0 \\
\hline Total & & & 80 & 30 & 43 \\
\hline
\end{tabular}

Tableau 4 : Jublains, "Impasse Romaine » : phase IV : répertoire de la céramique sigillée.

Tableau 4: Phase IV: shapes index of Terra sigillata.

ment à lèvre en poulie asymétrique (fig. $9, \mathrm{n}^{\circ} 1$ et 2 ). Des coupes à pâte orangée avec $\left(n^{\circ} 3\right)$ ou sans collerette $\left(n^{\circ} 4\right)$, parfois micacées $\left(\mathrm{n}^{\circ} 5\right)$ figurent également dans ce lot. Plusieurs mortiers sont également attestés, dont un exemplaire d'environ $35 \mathrm{~cm}$ de diamètre ( $\left.{ }^{\circ} 6\right)$. Parmi les amphores se distingue un conteneur Gauloise 12, à la pâte beige-orangé recelant de nombreuses inclusions de glauconie $\left(\mathrm{n}^{\circ} 7\right)$.

La céramique commune sombre se compose majoritairement de pots à lèvre anguleuse (fig. $9, n^{\circ} 8$ et 9) et d'un unique vase $\left(\mathrm{n}^{\circ} 10\right)$ proche des productions du site de $\mathrm{La}$ Bosse dans la Sarthe (Guillier, 1997).

Quelques exemplaires de céramique modelée (fig. 9, $\mathrm{n}^{\circ} 11$ à 13) complètent cet ensemble : une écuelle tripode et deux vases à lèvre plus ou moins marquée. Leur présence, tout au long de l'Antiquité, au sein des vaisseliers diablintes (Chuniaud et Mortreau, 2002, p. 174) et cénomans est aujourd'hui bien connue (Guillier, 1995b).
Mentionnons enfin la présence, comme en phase III, de quelques " palets » de jeu. Ils sont taillés dans des tuiles (fig. 9, $\mathrm{n}^{\circ} 14$ à 17), dans du schiste ( $\left.\mathrm{n}^{\circ} 18\right)$ ou du schiste ardoisier $\left(n^{\circ} 19\right)$. Leurs diamètres s'échelonnent de 57 à $81 \mathrm{~mm}$.

\section{Un mobilier exceptionnel : le trébuchet et le dodécaèdre}

Ces céramiques, pour importantes (dans le domaine chronologique) et intéressantes qu'elles soient, ne constituent toutefois pas des découvertes exceptionnelles. Il en va tout autrement des deux objets en alliage cuivreux (bronze?) issus de la couche de destruction de ce petit bâtiment sur sous-sol que sont un trébuchet et un dodécaèdre.

\section{Le trébuchet et ses graduations}

Complet, l'exemplaire de Jublains est constitué d'un fléau et de ses deux plateaux (fig. 10). Cet instrument de pesage est bien connu en contexte gallo-romain (Fauduet, $1992, \mathrm{n}^{\circ} 1017$ p. 138 et 139 ; Boucher et al., 1980, n 391 p. 81). Les plateaux sont en forme de " carapace de tortue " et mesurent $59 \times 52 \times 16 \mathrm{~mm}$ pour celui qui est complet et $49 \times 54 \times 17 \mathrm{~mm}$ pour l'autre; ils pèsent respectivement 12 et 11 gr. Leurs rebords très légèrement épaissis, repliés à l'intérieur et martelés, forment sur la surface interne une ligne irrégulière. Ils sont percés de trois trous de $2 \mathrm{~mm}$ de diamètre, disposés en triangle et postérieurs à la pliure. Le plateau complet présente une capacité de $20 \mathrm{~cm}^{3}$ (au ras du plateau) et de $36 \mathrm{~cm}^{3}$ " en tas".

Le fléau, long de $225 \mathrm{~mm}$, est intact et pèse $20 \mathrm{gr}$. Il est formé d'une tige de section circulaire $(5 \mathrm{~mm}$ au centre et 2,25 $\mathrm{mm}$ aux extrémités), légèrement cintrée et munie en son milieu d'une petite bélière présentant une lumière de $1 \mathrm{~mm}$ de diamètre; les longueurs des deux bras sont identiques. Les extrémités du fléau sont munies chacune d'un petit trou de suspension et adornées d'une petite boule terminale. Lors de la découverte du fléau, chacun des anneaux de suspension présentait la trace d'un fil de cuivre ou de bronze très fin, vraisemblablement destiné à recevoir les fils de suspension des plateaux, en matière périssable.

Un bras du fléau présente sur sa partie supérieure douze petites incrustations en argent de $0,5 \mathrm{~mm}$ de diamètre environ, légèrement oxydées (une est manquante). La première incrustation est placée au dessus du trou de suspension du plateau et les onze autres sont régulièrement espacées, d'un peu moins de $9 \mathrm{~mm}$, la dernière étant localisée à environ $9 \mathrm{~mm}$ de la lumière de la bélière. Sur une longueur de $110 \mathrm{~mm}$, à partir du milieu de la bélière et jusqu'au milieu du trou de suspension du plateau, ce bras est ainsi divisé en 12 intervalles réguliers de $9,16 \mathrm{~mm}$ chacun. Ces mesures correspondent assez précisément à un demi-doigt 


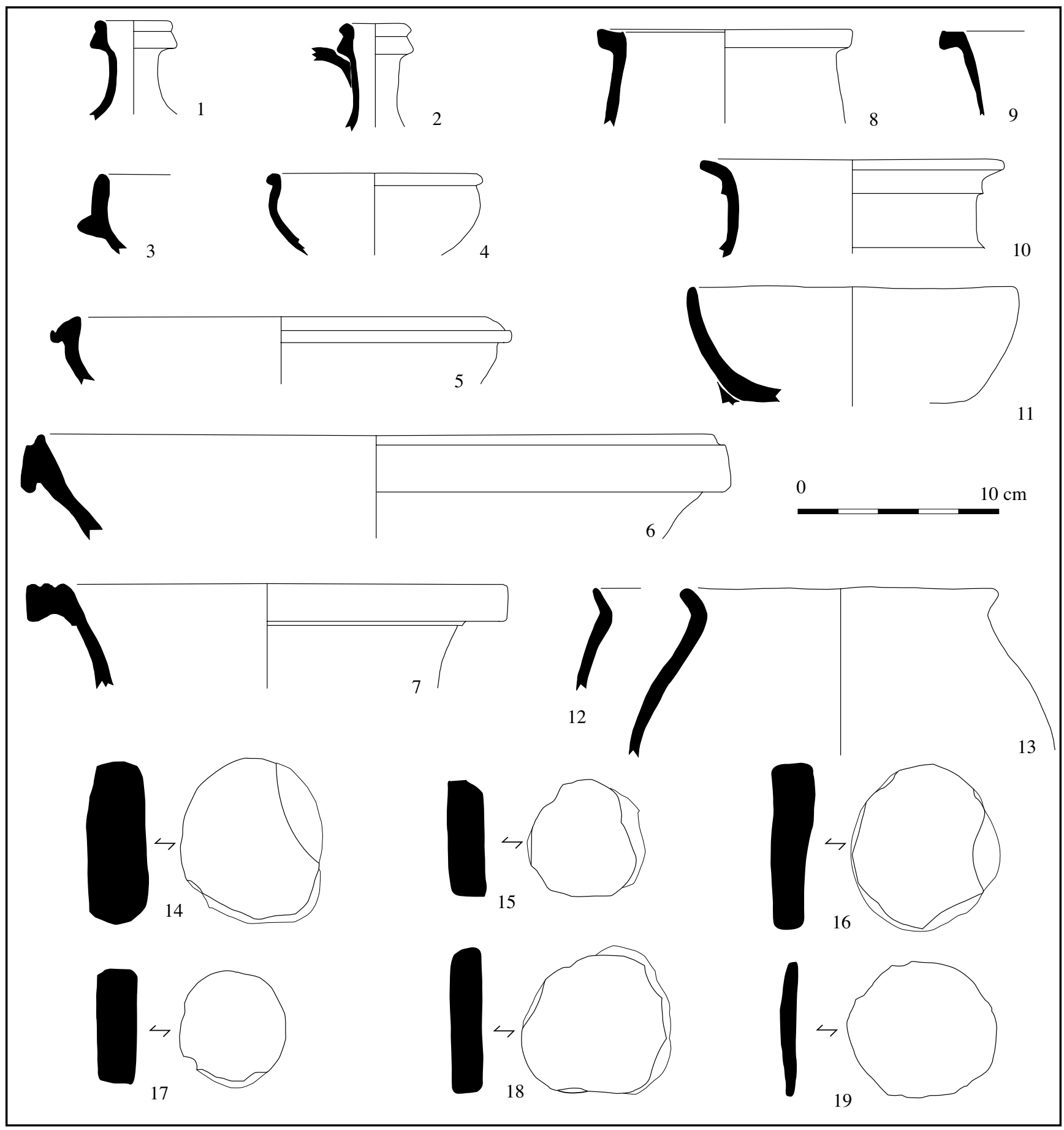

Figure 9 : Jublains, "Impasse Romaine » : le mobilier de la phase IV (suite) poteries et palets. Figure 9: Assemblage of Phase IV (continued): vessels and pucks.

(semi-digitus ou $1 / 32^{\mathrm{e}}$ de pied), la longueur du doigt étant ici de $18,33 \mathrm{~mm}$ pour une mesure théorique de $18,56 \mathrm{~mm}$ (Feugère, 1995; Santrot, 1996, p. 308). Enfin, la longueur de ce fléau est assez proche de $3 / 4$ de pied (ou 3 paumes).

Régionalement ce genre d'objet est assez peu commun. Mentionnons l'existence d'un important fragment de fléau de trébuchet découvert au Mans (Sarthe) dans les années $1960^{12}$. Son bras complet offre 12 petites entailles limitant des intervalles réguliers correspondant chacun à un demi-

12. Objet de l'ancienne collection M. Mémin au Mans, conservé actuellement aux musées du Mans (inv. n 2259). 


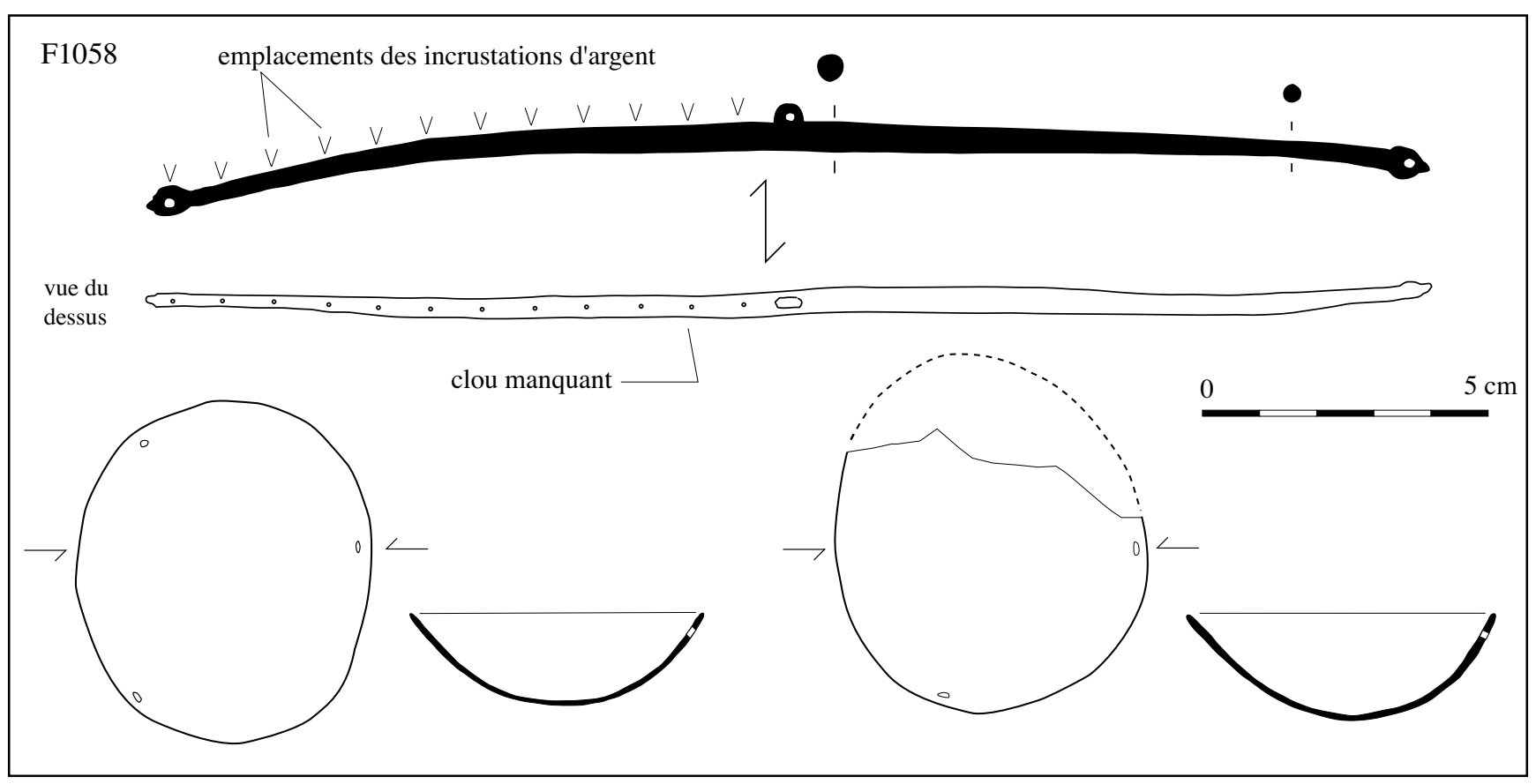

Figure 10 : Jublains, «Impasse Romaine » : le trébuchet découvert dans le petit bâtiment sur sous-sol (couche F1058) : le fléau et ses deux plateaux.

Figure 10: The balance uncovered in the small building with cellar (F1058 level): beam and pans.

pouce (semi-pollex ou 1/24 e de pied : 12,2 $\mathrm{mm}$ ); l'autre bras est, comme à Jublains, sans marque. Par comparaison, le trébuchet du Mans devait à l'origine mesurer un pied de long (296 mm). Un important fragment de trébuchet comparable à celui de Jublains a été découvert sur le site des Bolards en Côte-d'Or (Sautot, 1977, pl. XXV, n 4); sa longueur d'origine est estimée à $216 \mathrm{~mm}$. Sur un de ses bras ont été gravées onze incisions, rythmant des intervalles réguliers d'un peu moins de $9 \mathrm{~mm}$. Sur ce même site des Bolards, le fragment d'un fléau d'un autre trébuchet présente sur sa partie supérieure des ocelles distantes de 12 à 13 mm (Sautot, 1977, pl. XXV, n 6). Deux fléaux de trébuchets, longs de 260 et $113 \mathrm{~mm}$ et découverts sur la villa de Bapteste à Moncrabeau dans le Lot-et-Garonne, sont conservés au musée de Nérac. Enfin, mentionnons des modèles assez comparables provenant d'Italie : Modène, Luni ou Pompeï, mais aussi de Zeugma en Turquie, où le fléau découvert dans la « maison de Poséidon ${ }^{13}$ ", long de $287 \mathrm{~mm}$, possède deux systèmes de graduation différents (informations $M$. Feugère).

Les graduations visibles sur ces trébuchets (souvent sur un seul bras), offrant des intervalles exprimés en semi-digiti (environ $9 \mathrm{~mm}$ ) ou en semi-pollices (12 à $13 \mathrm{~mm}$ ), impliquent l'existence d'un petit peson coulissant ou curseur (que

13. Dans le péristyle P9, dans une couche de destruction datée du milieu du III ${ }^{e}$ siècle. nous n'avons pas retrouvé à Jublains) permettant d'affiner les pesées par de petits équilibrages (Di Pasquale, 2001, p. 283).

Ce genre d'objet présente un aspect fortement utilitaire, lié au commerce et à l'artisanat. Il permet de vérifier l'aloi d'une monnaie ou bien encore de peser des matières précieuses (métaux, épices...), sa capacité ne devant pas excéder une livre $(327,45 \mathrm{~g})$. Il n'en présente pas moins une certaine charge symbolique ${ }^{14}$.

\section{Le dodécaèdre}

Cet objet, intact malgré une fêlure à peine visible sur une arête, est haut de $59 \mathrm{~mm}$ (posé) mais seulement de 48 à $52 \mathrm{~mm}$ de face à face; son diamètre maximum est de $74 \mathrm{~mm}$ et il pèse $81 \mathrm{gr}$ (fig. 11). Il semble avoir été coulé à la cire perdue puis soigneusement rectifié. Il présente douze faces pentagonales de $21 \mathrm{~mm}$ d'arête, pourvues pour dix d'entre elles d'une ouverture circulaire, soulignée de cercles concentriques gravés dans le métal. Ces ouvertures offrent des diamètres variant de 10,5 à $22 \mathrm{~mm}$. Les deux autres faces, dépourvues de cercles concentriques, présentent des

14. La balance est utilisée pour peser le bien et le mal, elle est aussi un attribut de la Justice (pour la pesée des âmes et ce, depuis la plus haute Antiquité). Elle est un des symboles de Mercure; chez les chrétiens ce symbole est passé à saint Michel. D'autre part, la balance est liée à l'un des signes du Zodiaque : septembre-octobre (Hall, 1994, p. 71). 


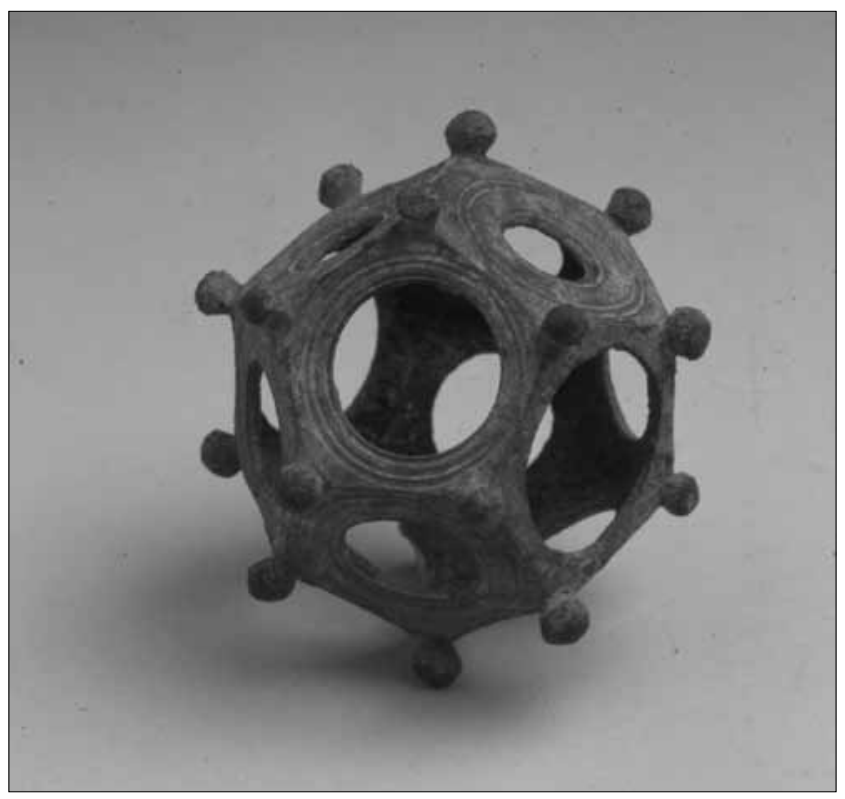

Figure 11 : Jublains, "Impasse Romaine » : le dodécaèdre provenant de la couche de destruction F1058 du petit bâtiment sur sous-sol (cliché A. Szczuczynski).

Figure 11: The dodecahedron from destruction level F1058 in the small building with cellar.

ouvertures ovales $(21 \times 26 \mathrm{~mm})$, elles sont placées en opposition sur l'objet, y matérialisant éventuellement un " haut " et un "bas". Chaque sommet, à la jonction de trois faces, est agrémenté d'une petite boule de 5 à $6 \mathrm{~mm}$ de diamètre environ, soudée au corps même du dodécaèdre.

Parmi les objets les plus comparables à celui de Jublains, citons ceux de Reims, d'Avenches (Suisse) et de Feldberg (Allemagne). Nous n'en présentons pas un dessin purement archéologique mais un double rabattement (fig. 12), effectué selon les préconisations de Paul-Marie Duval (1981), permettant de comparer les différents objets sur une même base descriptive.

Il n'entre évidemment pas dans le cadre de cette contribution de présenter un état de la recherche sur ce type d'objet ${ }^{15}$ et nous renvoyons aux synthèses publiées. En français, celles de J. de Saint-Venant (1907) et de W. Déonna (1954), bien qu'assez anciennes, n'en sont pas moins détaillées et bien documentées. Un article récent, reprenant les données sur cet objet, conclut qu'en l'état actuel de la recherche rien ne permet d'en déterminer la fonction (Nouwen, 1994). Un rapide panorama des lieux de découvertes et des questions chronologiques et fonctionnelles s'impose toutefois, afin de

15. Nous ne présentons ici que les références bibliographiques les plus indispensables à notre propos; en effet, il existe bien plus d'articles ou de notes sur ce sujet que d'objets découverts! discuter des interprétations possibles de cette découverte exceptionnelle.

Actuellement, sur environ une centaine de dodécaèdres inventoriés (Guggenberger, 1999), une trentaine proviennent de France. Les autres ont été mis au jour en Autriche, en Belgique, en Grande-Bretagne, en Hongrie, aux Pays-Bas, en Suisse, dans l'ex-Yougoslavie et surtout en Allemagne.

En France, d'après les éléments à notre disposition, nous observons une concentration de ces dodécaèdres en Gaule Belgique et dans le sud de la Gaule Lyonnaise (Bourgogne, ouest de la Franche-Comté et nord de Rhône-Alpes). La moitié sud de la France est très peu représentée (Bordeaux et Arles), de même pour le Grand-Ouest (Poitiers, Rouen et Jublains). Les auteurs consultés s'accordent pour noter leur absence de l'espace circum-méditerranéen (Italie, Grèce, Orient, Espagne ou Afrique du nord). Par contre, les trouvailles se répartissent très nettement sur l'ancien territoire celtique, alors que la forme "dodécaèdre " se situerait pour la période antique dans les conceptions pythagoricienne et platonicienne de l'univers (Saint-Michel, 1951; Déonna, 1954).

Les contextes des découvertes de ces dodécaèdres sont rarement mentionnés, celles-ci étant le plus souvent anciennes. Nous avons relevé cinq sites militaires, deux thermes, un théâtre, une tombe, un trésor monétaire du Iv siècle, le lit d'un fleuve en Hollande et le comblement d'un puits.

$\mathrm{Si}$, pour la plupart des exemplaires connus, les contextes archéologiques sont incertains, les éléments de datation ne le sont pas moins. Toutefois, dès 1907, J. de Saint-Venant émet l'hypothèse d'une datation assez tardive : $\mathrm{III}^{\mathrm{e}}$ et IV $\mathrm{IV}^{\mathrm{e}}$ siècles; celle-ci est confirmée par W. Déonna (1954) et par d'autres chercheurs, dont F. Benoît (1957). Plus récemment P.-M. Duval (1981) considère qu'ils auraient pu être parfois fabriqués dès le $\mathrm{II}^{\mathrm{e}}$ siècle. En ce qui concerne l'objet de Jublains, il y a tout lieu de croire qu'il a été utilisé au cours de la première moitié du III siècle (cf. supra).

Les interprétations les plus diverses (voire les plus fantaisistes) ont été émises au sujet de ces objets, et ce dès le moment où les premiers antiquaires s'y sont intéressés, sous Louis XV! (de Caylus, 1752-1767). Nous avons relevé les hypothèses suivantes : instruments d'arpentage, aspersoirs, lampes, gabarits de bijoutiers, mesures, garnitures de goupillons, calibres à flans monétaires, jouets ou bilboquets, pommeaux de sceptres, dés de jeux, chandeliers à calibres multiples, chefs d'œuvre de maîtrise ou encore élément d'illustration des théories pythagoriciennes (Saint-Venant 1907; Saint-Michel, 1951; Déonna, 1954; Nouwen, 1994). S'opposent ainsi deux types de conceptions radicalement différentes : celles que nous pouvons qualifier de purement utilitaires et celles, plus chargées de symbolique, à mettre en rapport avec le pythagorisme romain (Déonna, 1954, p. 67) et la mystique des nombres 


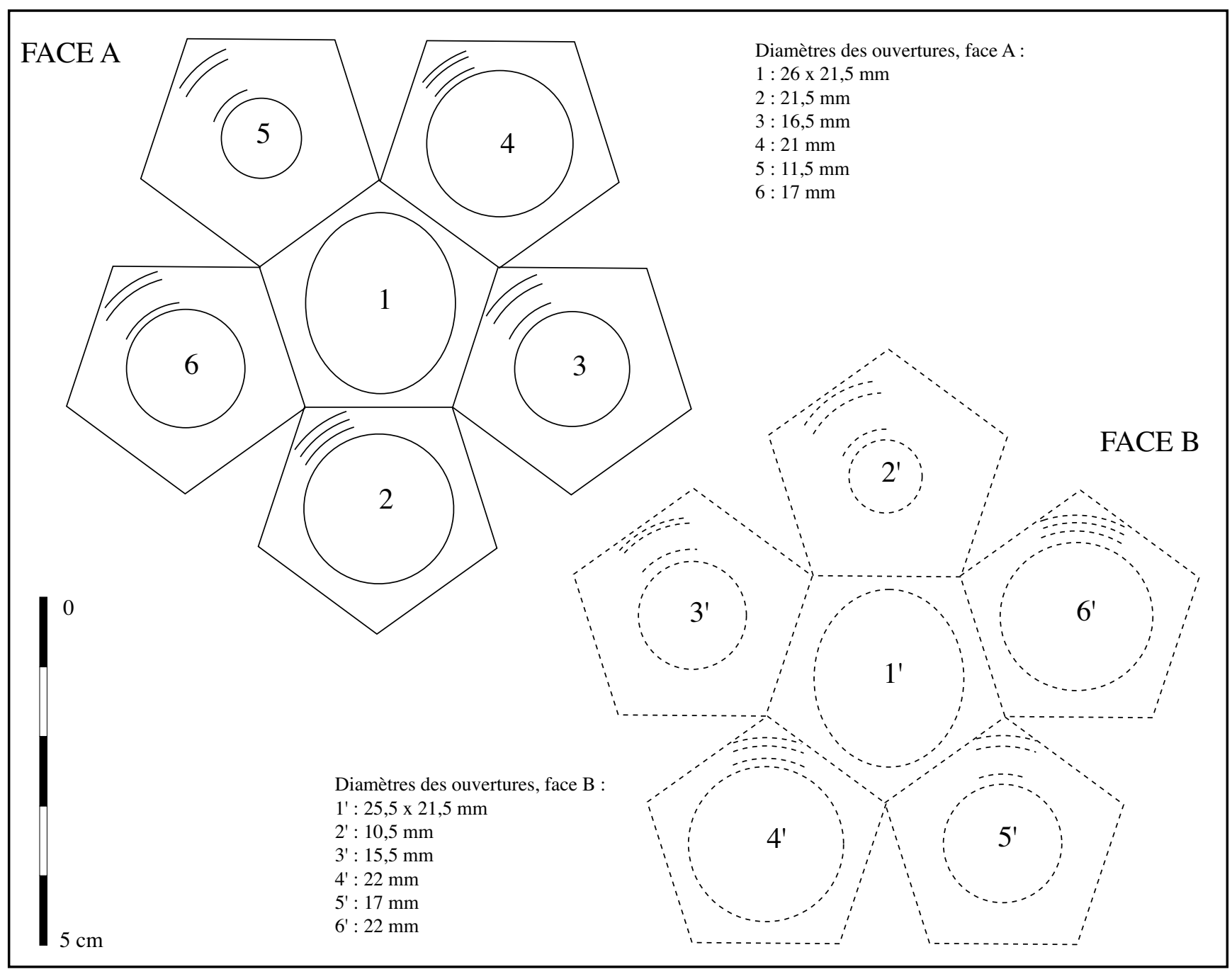

Figure 12 : Jublains, «Impasse Romaine » : le dodécaèdre, rabattement des 12 faces. Figure 12: The Jublains dodecahedron: rebatement of the 12 faces.

(les 12 signes zodiacaux... : Thévenot, 1955). Ces dernières trouvent une application dans des actes de divination ou de consultation par le sort, puisque ces dodécaèdres symboliseraient l'univers ou le ciel. La découverte récente à Genève (Suisse) d'un dodécaèdre plein, en plomb revêtu d'argent et portant gravé sur chacune de ses faces un signe du Zodiaque (Cervi-Brunier, 1985) relance le débat. Enfin, signalons qu’au $\mathrm{XVI}^{\mathrm{e}}$ siècle, dans le "Plaisant jeu du dodécaèdre de Fortune " ce type d'objet servait de dé pour des jeux de divination (Déonna, 1954, p. 89).

\section{DE L'ANTIQUITÉ TARDIVE À L'ÉPOQUE MODERNE}

Après le milieu du III $^{\mathrm{e}}$ siècle, nombre d'aménagements antiques de Jublains sont détruits ou récupérés, tels le mur nord des thermes ou les murs est et sud de la structure excavée. Les témoignages archéologiques de ces évènements sont peu nombreux; relevons une monnaie de Tétricus (Annexe, M14) et de rares tessons dont un petit fragment attribuable à une lèvre d'un vase dit "à baguette ", connu par ailleurs à Jublains (Bocquet et al., 2004, fig. 26, vase $\mathrm{n}^{\circ}$ 9). Un des ateliers de fabrication de ce type de vaisselle a été mis au jour dans la Sarthe (Guillier, 1997), pour une production au III ${ }^{\mathrm{e}}$ siècle (Delage et Guillier, 1997). Quelques tessons à la pâte grise sableuse soulignent une maigre occupation au 
$\mathrm{IX}^{\mathrm{e}}$ siècle, tandis que deux fossés comblés de pierres constituent de probables drains de la période moderne (par exemple F1014, fig. 4, coupe 1).

\section{7. ÉLÉMENTS DE CONCLUSION}

Cette fouille archéologique au cœur même de l'antique Nouiodunum, immédiatement au nord des thermes antiques, a, malgré sa faible surface, permis la collecte d'informations qui confortent des données acquises par ailleurs, tout en offrant matière à quelques nouveautés des plus intéressantes.

En premier lieu, elles concernent le vaste creusement bordant à l'ouest la rue $\mathrm{A}$, observé par nous et formant notre phase I (fig. 3) et précédemment noté par Jacques Naveau (1992, fig. $28 ; 1997$, p. 79). Il s'agit du témoin privilégié d'un premier état de voirie, tibéro-claudien vraisemblablement, dont les matériaux auraient été totalement récupérés tandis que l'on déplaçait la rue vers l'est sous le règne de Néron pour former la nouvelle rue A. La mise en place de ce premier état de voirie pré-flavien, déjà observé à Jublains par exemple à la Grande-Boissière (Bocquet et al., 2004), a jeté les bases d'une orientation qui ne s'est vraiment figée que plus tard, avec le plan flavien mis en évidence par Jacques Naveau (1986). Quelques grands monuments témoignent déjà sous Claude de la force de ce premier état de voirie préflavien, notamment le forum.

En deuxième lieu, cette petite fouille a permis de reconnấtre la constitution de la rue 8 et de ses abords, rue qui borde au nord les thermes antiques. Elle ne se distingue guère morphologiquement de celles observées précédemment (Naveau, 1992, p. $46-47$ et 1997, p. 84-87; Bocquet et al., 2004). La chaussée est formée de couches de blocs de granite et d'arène granitique compactée ou de scories de fer. La limite nord de cette rue était marquée par un muret bordant très probablement un trottoir (avec portique?), séparé de la chaussée par un caniveau. Sa limite sud est moins bien connue. Par ailleurs, la découverte d'une monnaie de Néron sous le premier remblai de voie, permet de confirmer l'hypothèse chronologique de l'installation de la trame viaire de Nouiodunum, telle qu'elle apparait au début de la période flavienne.

Mais c'est surtout la présence d'un petit bâtiment sur soussol excavé, occupé lors de la première moitié du III ${ }^{\mathrm{e}}$ siècle, et des mobiliers qui lui sont associés, qui a le plus retenu notre attention avec la mise au jour d'un trébuchet constitué d'un fléau et de ses deux plateaux, et d'un dodécaèdre en bronze à angles bouletés. Le premier devait servir à la pesée fine de matières précieuses ou semi-précieuses; la fonction du second reste en revanche délicate à déterminer même si la fouille de Jublains peut apporter quelque éclairage. Le mobilier céramique qui accompagne ces objets comporterait une plus grande proportion de vases fermés (bouteille, cruche, pots ovoïdes) et une moindre présence des vases de cuisine, par rapport à d'autres lots contemporains.

L'ensemble des informations recueillies tend à montrer que ce bâtiment ne correspondait pas à un espace privé ordinaire. Sa situation à l'ombre d'un grand bâtiment public, à l'intersection de deux axes essentiels de la ville (Cardo A et Décumanus 8 ), probablement très passagers, constitue une position idéale pour une implantation commerciale.

En fait, suivant que l'on accorde une importance plus grande au trébuchet ou au dodécaèdre, nous pourrions être en présence d'une échoppe de commerçant ou d'artisan (changeur, orfevre, bronzier, marchand d'épices ou de matières précieuses - onguents ou liquides conservés dans les vases fermés) ou encore de l'antre d'un devin ou d'une voyante (les deux n'étant d'ailleurs pas incompatibles...).

\section{AnneXe}

\section{Les monnaies du site de l' "Impasse Romaine » à Jublains (Mayenne)}

Paul-André Besombes

\section{Abréviations utilisées}

Crawford: Crawford, M. H., 1974 - Roman Republican Coinage, Cambridge.

Giard: Giard, J.-B., 1988 - Catalogue des monnaies de l'Empire romain, I Auguste, Paris, Bibliothèque nationale, $260 \mathrm{p}$.

J. Van Heesch : Van Heesch, J., 1993 - Proposition d'une nouvelle datation des monnaies en bronze à l'autel de Lyon frappées sous Auguste. Bulletin de la Société française de Numismatique, 4, p. 535-538.

$\boldsymbol{R I C}$ : Sutherland, C. H. V., 1984 - The Roman Imperial Coinage : from 31 to $A D$ 69. Londres; Mattingly, H., et Sydenham, E. A., 1926 - The Roman Imperial Coinage, Vespasian to Hadrian. Londres.

Elmer : Elmer, G., 1941 - Die Münzprägung der gallischen Kaiser in Köln, Trier und Mainland. Bonner Jahrbücher, 146, Darmstadt, p. 1-106.

\section{République romaine}

M1 - M. AEMILIUS SCAURUS, P. HYPSAEUS : Denier (argent, 2,99 g., 8h)

$\mathrm{D} /$ Chameau à droite, devant personnage assis; au dessus $[\mathrm{M}$ SC]AVR/[A]ED CVR, de part et d'autre [EX S C], dessous [REX] ARET[AS]. 
$\mathrm{R} /$ Jupiter dans un quadrige à gauche; au dessus [C] HYPSAE[VS]/AED [CVR]; dessous [C] HYPSAE[COS]/ PREIVE.

Crawford $4221 \mathrm{~b}$ : Rome 58 avant J.-C.

Forte usure; les deniers républicains ont été retirés de la circulation sous Trajan (98-117); US 1000.

\section{Empire romain}

\section{Julio-claudiens}

M2 - AUGUSTE (27 avant J.-C.-14 après) : AS (cuivre, 10,19 g., 5h)

$\mathrm{D} /[\mathrm{CA}] \mathrm{ES}[\mathrm{AR}][\mathrm{PONT}] \mathrm{MA}[\mathrm{X}]$; tête laurée à droite.

R/ [ROM ET AVG] ; l'autel de Lyon.

RIC 230, Giard 73 ou 117, J. Van Heesch : Lyon 8/7-3 avant J.-C.

Usure marquée; US 1074.

M3 - AUGUSTE (27 avant J.-C.-14 après) pour Tibère César : As (cuivre, 10,49 g., 12h)

D/ [TI CAESAR AVGVST F IMPERAT VII] ; tête laurée de Tibère à droite.

R/ [ROM ET AVG] ; l'autel de Lyon.

RIC 245, Giard 114 : Lyon 12 après J.-C. - début du règne de Tibère (?).

Usure marquée; US 1079.

M4 - CLAUDE (41-54) : Dupondius (orichalque, 9,99 g.; 6h)

D/ [TI CLAVD]IVS CAESAR AVG P M TR P [IMP]; tête nue à gauche.

R/ CONSTANTIAE AVGVSTI; Constantia, casquée et en Habit militaire, debout à gauche, main droite levée et tenant une longue lance.

RIC : Rome 41-50 (?).

Faible usure, US 1035.

M5 - CLAUDE (41-54) : As (cuivre, 6,88 g., -)

D/ [TI CLAVD]IVS [CA]ESAR [AVG P M TR P IM]P; tête nue à gauche.

$\mathrm{R} /$ Illisible.

RIC - atelier non officiel.

US 1064.

M6 - CLAUDE (41-54) : As (cuivre, 8,81 g., -)

$\mathrm{D} /$ Tête nue de Claude à gauche.

$\mathrm{R} /$ Illisible.

RIC - atelier non officiel.

Forte usure; US 1064.

M7 - NERON (54-68) : As (cuivre, 10,01 g., 9h)

D/ [N]ERO [CAESAR AVG G]ERM IMP; tête nue à droite.

R/ [PACE P R V]BIQ [PARTA IANVM CLVSIT] [S] C; le temple de Janus à Rome.

RIC 306 : Rome 64.
Faible usure; US 1079.

M8 - NERON (54-68) : As (cuivre, 7,59 g., 6h)

D/ NERO CAESAR AVG GERM IMP; tête laurée à droite.

$\mathrm{R} / \mathrm{S} \mathrm{C}$; Victoire volant à droite, tenant des deux mains un bouclier où il est inscrit SPQR.

RIC 312, Rome 64.

Faible usure; US 1063.

M9 - NERON (54-68) : As (cuivre, 11,09 g., 6h)

$\mathrm{D} /$ tête de Néron à droite.

R/ PON[TIF MAXIM TR POT I]M[P] [S] C; Néron avançant à droite, vêtu en Apollon citharède, jouant de la lyre. RIC -.

Faible usure malgré une forte corrosion; US 1035.

\section{Flaviens}

M10 - VESPASIEN (69-79) pour TITUS César : As (cuivre, 8,78 g., -)

D/ [T] CAESAR...; tête laurée à droite.

$\mathrm{R} /$ Illisible.

RIC -, Lyon 76-78.

Usure marquée, US 1065.

M11 - DOMITIEN (81-96) : As (cuivre, 8,50 g., 6h)

D/ IMP [.....] AUG [.....]; tête de Domitien laurée à droite.

$\mathrm{R} /$ personnage féminin debout à gauche, tenant la corne d'abondance et une balance (?) : Moneta (?).

RIC -, Rome.

Forte usure; US 1020.

\section{Antonins}

M12 - TRAJAN (98-117) : Dupondius (orichalque, 10,76 g., 6h)

D/ IMP ......; tête de Trajan à droite.

$\mathrm{R} / \mathrm{S} \mathrm{C}$ à l'exergue; personnage assis (?).

RIC - atelier non officiel (?) début du règne.

Forte usure; US 1016.

M13 - HADRIEN (117-138) : Dupondius (orichalque, 9,48 g., 6h)

D/ HA[DRIANVS] A[VGVSTVS]; tête nue à droite.

$\mathrm{R} /[\mathrm{COS} \mathrm{I}] \mathrm{II} \mathrm{P}[\mathrm{P}][\mathrm{S}] \mathrm{C}$; Rome debout à droite le pied sur un casque, tenant une lance et une corne d'abondance.

RIC 716 : Rome 132-134.

Usure marquée, US 1058.

\section{Empire Gaulois}

M14 - TETRICUS (271-274) pour TETRICUS II César : Antoninien (billon, 3,18 g., 6h)

D/ [C PIVS ESV TE]TRICVS [CAES] ; tête radiée de Tétricus II à droite.

R/ Personnage debout : Spes (?).

Elmer 791 et suivants : 273-274.

US 1010 . 


\section{Remerciements}

Ils vont à M. Piton (fouille et collaboration au DFS) et $N$. Fouillet (fouille), à D. Fillon (topographe), à la mairie de Jublains pour son aide technique, à M. Mortreau pour ses conseils, à M. Feugère pour ses renseignements sur Zeugma ainsi qu'à J. Naveau et A. Bocquet pour leur aide.

\section{Bibliographie}

BENoîT, F., 1957 - Deux énigmes archéologiques : dodécaèdre perlé d'Arles et anneau octogonal bouleté de Vichy, OGAM, 9, p. 104-114, fig. 2-8 et pl. IX-XII.

Bocquet, A., Chuniaud, K. et Naveau, J., 2004 - Le quartier antique de la Grande-Boissière à Jublains (Mayenne), Revue archéologique de l'Ouest, 21, p. 131-174.

Boissel, R. et DieHL, R., 1972 - La nécropole gallo-romaine méridionale de Noeodunum (Jublains) (prospections de 1970 et 1971), Bulletin de la Commission historique et archéologique de la Mayenne, 25 (242), p. 3-52.

Boucher, S., Perdu, G. et Feugère, M., 1980 - Bronzes antiques, II, Instrumentum, aegyptica. Lyon, Musée de la civilisation gallo-romaine, $135 \mathrm{p}$.

Bouvet, J.-Ph., 2001 - La Sarthe 72. Carte Archéologique de la Gaule, Paris, Académie des Inscriptions et Belles-Lettres, 519 p.

DE CaYlus, A. C. P., 1752-1767 - Recueil d'antiquités égyptiennes, étrusques, grecques et romaines. Paris, Dessaint et Saillant, Vol. III, pl. CXI, 3, 4.

Cervi-Brunier, I., 1985 - Le dodécaèdre en argent trouvé à Saint-Pierre de Genève. Revue suisse d'Art et d'Archéologie, 42, p. 153-156.

Chuniaud, K. et Mortreau, M., 2002 - Un ensemble céramique de la première moitié du $\mathrm{II}^{\mathrm{e}}$ siècle sur le site de La GrandeBoissière à Jublains (Mayenne), SFECAG (Actes du congrès de Bayeux), p. 159-175.

Coffineau, E. et Dubant, D., 2002 - La céramique commune du site de la plaine d'Hermesnil (Seine-Maritime) de la seconde moitié du II $^{\mathrm{e}}$ siècle de notre ère, SFECAG (Actes du Congrès de Bayeux), p. 131-140.

Delage, R. et Guillier, G., 1997 - La céramique confrontée au problème de la datation des niveaux du III $^{\mathrm{e}}$ siècle : quatre exemples Manceaux (Sarthe), SFECAG (Actes du Congrès du Mans), p. 255-278.

DÉonNA, W., 1954 - Les dodécaèdres gallo-romains en bronze ajourés et bouletés. À propos du dodécaèdre d'Avenches. Bulletin de l'Association Pro Aventico, 16, p. 18-89, 18 fig., 1 pl.

Di Pasquale, G., 2001 - Les instruments de pesée. Pompéi. Nature, sciences et techniques (Catalogue d'exposition, Palais de la Découverte), Milan, Electa, p. 283-285.
Duval, P.-M., 1981 - Comment décrire les dodécaèdres galloromains en vue d'une étude comparée, Gallia, 39, p. 195200.

Fauduet, I., 1992 - Musées d'Évreux, Bronzes gallo-romains, Instrumentum, Evreux, Musées d'Évreux, 171 p.

Ferrette, R., 2003 - La céramique gallo-romaine du site de Monterfil II à Corseul (Côtes-d'Armor). Études d'ensembles de l'époque augustéenne au début du IV siècle, Montagnac, éd. Monique Mergoil, 224 p.

Feugère, M., 1995 - Une mesure d'un demi-pied romain à Châlon-sur-Saône (Saône-et-Loire), Revue archéologique de l'Est, 46, p. 151-153.

Gugenberger, M., 1999 - Die römischen Dodekaeder. Eine Gesamtdarstellung (Mémoire de master), Innsbruck, université Leopold-Franzens, 230 p.

GuILliER, G., 1995a - Jublains "Impasse Romaine ». 53.122.145 AH (Mayenne) (DFS de sauvetage urgent), AFAN/Service régional de l'Archéologie des Pays-de-la-Loire, 57 p., 26 pl., annexes.

Guillier, G., 1995b - Un aspect méconnu de la céramique du Haut-Empire : la vaisselle modelée, l'exemple du Mans (Sarthe), SFECAG, (Actes du congrès de Rouen), p. 217-233.

Guillier, G., 1997 - La production céramique du Haut-Empire de l'officine rurale de La Bosse (Sarthe), SFECAG, (Actes du Congrès du Mans), 1997, p. 239-254.

HaLl, J., 1994 - Dictionnaire des mythes et des symboles, Aubenas, éd. Gérard Monfort, 416 p.

JARDREL, K., 2002 - Le mobilier céramique du III ${ }^{\mathrm{e}}$ siècle issu du site "Les Préaux " à Vieux (Calvados), SFECAG (Actes du Congrès de Bayeux), p. 141-158.

LE Goff, E., 2003 - «Armorique » et " Bretagne » : réflexion sur l'assimilation de ces concepts pour la fin de l'Âge du Fer, in Mandy, B. et de Saulce, A. (dir.), Les Marges de l'Armorique à l'Âge du Fer, Archéologie et Histoire : culture matérielle et sources écrites (Actes du XXIII' colloque de l'AFEAF), Revue archéologique de l'Ouest, supplément n 10, p. 103-117.

Moireau, F., 1992 - Le dépotoir des "Murgets ", à Tavers (Loiret) : étude de la céramique, Revue archéologique du Centre de la France, 31, p. 177-188.

Naveau, J., 1986 - Le plan antique de Jublains (Mayenne), Revue archéologique de l'Ouest, 3, p. 107-117.

Naveau, J., 1992 - La Mayenne 53. Carte Archéologique de la Gaule, Paris, Académie des Inscriptions et Belles-Lettres, $176 \mathrm{p}$.

Naveau, J., (dir.) 1997 - Recherches sur Jublains (Mayenne) et sur la cité des Diablintes, Rennes, éd. RAO, coll. "Documents archéologiques de l'Ouest ", $352 \mathrm{p}$.

Nouwen, P., 1994 - Les dodécaèdres gallo-romains ajourés et bouletés. Histoire et problèmes, Bulletin de L'Institut archéologique liégeois, 56, p. 85-108. 
Philippe, J., Jobelot, N. et Vermeersch, D., 1996 - Santeuil : Les Épagnes, in Jobelot, N. et Vermeersch, D. (dir.), Archéologie en Val-d'Oise, 5: Céramiques gallo-romaines du Val-d'Oise, p. 114-139.

SAINT-Michel, L., 1951 - Situation des dodécaèdres celto-romains dans la tradition symbolique pythagoricienne. Bulletin de l'Association Guillaume Budé, 4 (2), suppl. 10, p. 92-116.

DE Saint-Venant, J., 1907 - Dodécaèdres perlés en bronze creux ajourés de l'époque gallo-romaine, Nevers, Mazerons frères, $54 \mathrm{p}$.
SAntrot, J., 1996 - Bronzes et fer de Dax, Landes : la cachette d'un « antiquaire-restaurateur " au Iv" siècle après J.-C. Gallia, 53, p. 251-343.

SaUtot, M.-C. 1977 - Une collection d'objets de bronze provenant des Bolards (Côte-d'Or). Revue archéologique de l'Est et $d u$ Centre-Est, 28, n ${ }^{\circ} 109-110$, p. 285-349.

Thévenot, E., 1955 - La mystique des nombres chez les Galloromains. Dodécaèdres Bouletés et taureaux tricornus, Revue archéologique de l'Est et du Centre-Est, 6, p. 291-295. 
